\title{
One-loop structure of the $U(1)$ gauge model on the truncated Heisenberg space
}

\author{
Maja Burića, Luka Nenadović ${ }^{\mathrm{b}}$, Dragan Prekrat ${ }^{\mathrm{c}}$ \\ Faculty of Physics, University of Belgrade, P.O. Box 44, 11001 Belgrade, Serbia
}

Received: 10 October 2016 / Accepted: 16 November 2016 / Published online: 5 December 2016

(C) The Author(s) 2016. This article is published with open access at Springerlink.com

\begin{abstract}
We calculate divergent one-loop corrections to the propagators of the $U(1)$ gauge theory on the truncated Heisenberg space, which is one of the extensions of the Grosse-Wulkenhaar model. The model is purely geometric, based on the Yang-Mills action; the corresponding gaugefixed theory is BRST invariant. We quantize perturbatively and, along with the usual wave-function and mass renormalizations, we find divergent nonlocal terms of the $\square^{-1}$ and $\square^{-2}$ type. We discuss the meaning of these terms and possible improvements of the model.
\end{abstract}

\section{Introduction}

Unsuccessful attempts to quantize the gravitational field by the usual methods indicate that the structure of spacetime at the Planck scale is very different from the classical one. Such a conclusion is also indicated by the existence of singularities in general relativity and divergences in perturbative quantum field theory. There are many reasons to believe that the theory of quantum gravity will not be local in the conventional sense [1]; however, locality is in the core of standard field theories and one has to solve many problems in order to formulate a consistent framework to describe nonlocal classical and quantum fields.

Perhaps the first idea of how to 'delocalize' points was the Kaluza-Klein extension of spacetime by additional compact dimensions. Alternatively, nonlocality can be introduced through the structure of elementary constituents as in string theory; in both cases the underlying spacetime is a Riemannian manifold. Another way to introduce nonlocality is to assume that spacetime is described by an algebra of noncommuting operators.

\footnotetext{
a e-mail: majab@ipb.ac.rs

be-mail: Inenadovic@ipb.ac.rs

c e-mail: dprekrat@ipb.ac.rs
}

We analyze this approach, presuming that noncommutativity of the coordinates of 'quantum spacetime' is physical; we further investigate properties of quantum fields defined on it. Our motivation for this study is twofold. The first is the expectation that the existing knowledge about operator algebras and their representations gives enough tools to build a compact mathematical framework. The second is the hope that the 'amount of nonlocality' introduced algebraically is restricted and that it can provide us with reasonable and interesting physics.

Mathematical and physical results which concern noncommutative geometry and noncommutative field theories are numerous. In a brief summary one can say that geometry and classical field theories are understood fairly well, whereas quantization is still an open problem. A technical explanation is that, though in quantization the effects of nonlocality improve the ultraviolet behavior of a theory, the mixing of large and small length scales induced by noncommutativity transfers divergences to the infrared sector (UV/IR mixing). This is clearly established for theories defined on the Moyal space. On the other hand, quantum field theories defined on spaces with finite matrix representations are finite, and thus they can be viewed as matrix regularizations of (the corresponding) commutative theories. Moreover, there are models in which the continuous limit of a matrix theory has better quantization properties than its commutative predecessor [2].

The first fully renormalizable theory formulated on a noncommutative space is the Grosse-Wulkenhaar (GW) model $[3,4]$. It describes a real scalar field on the Moyal space of Euclidean signature confined in the oscillator potential. The potential term induces symmetry between long and short distances, the so-called Langman-Szabo (LS) duality [5], which is roughly of the form $x \leftrightarrow p$. The field propagator is given by the Mehler kernel: it regularizes the UV behavior of the theory keeping the IR sector finite. Renormalizability of the GW model is established and thoroughly examined by vari- 
ous methods [6-9]; its exceptional mathematical properties induced a lot of subsequent work. Two renormalizable spinor field theories analogous to the GW model have been found: in the first one [10], the representation of spinors is chosen in such a way that the square of the propagator is the Mehler kernel. The other model, due to Vignes-Tourneret (VT) [11], is a noncommutative generalization of the Gross-Neveu model [12]. In both theories the Lagrangian has an explicit dependence on coordinates and breaks the translation invariance. To improve the last property an interesting translationally invariant model, which is also renormalizable, was proposed in [13]. It contains, instead of the oscillator potential, the $\square^{-1}$ term in the kinetic part of the action, thus introducing another version of the LS duality: $\square \leftrightarrow \square^{-1}$.

All attempts to find a renormalizable gauge model à la Grosse-Wulkenhaar have been unsuccessful till the present. Several strategies have been used, all mainly based on parallels with standard gauge theories. As most of the results are thoroughly reviewed in [14] we will here recall only some guiding ideas. The first proposal was to transform the gauge propagator into the Mehler kernel by nonlinear gauge fixing [15]; however, the appearance of the tadpole divergence made the theory nonrenormalizable. Another strategy was to impose the LS duality $[16,17]$ : the action for the gauge field was defined by minimal coupling to the GW scalar and subsequent integration of the scalar field. Although the obtained, induced theory has good symmetry properties (LS duality becomes invariance under the exchange $\left[x^{\mu},\right] \leftrightarrow\left\{x^{\mu},\right\}$ ), it does not have the trivial vacuum solution and perturbative quantization is not well defined. It is important to notice that the explicit coordinate dependence of the induced gauge theory can be elegantly rewritten using the covariant coordinates which were introduced much earlier [18]. For recent results on quantization of this theory in the matrix base we refer to [19].

The idea which we have developed in the previous papers is that specific forms of the GW and VT actions are due to the underlying (noncommutative) geometry. The idea is based on the result that the two-dimensional GW action can be viewed as an action defined on particular curved three-dimensional space after the Kaluza-Klein (KK) reduction [20]. The VT action, similarly, is the spinor action on the same space [21]. In both cases matter is nonminimally coupled to the background curvature and torsion. The employed approach gives also the $U$ (1) Yang-Mills (YM) theory which consists, after the KK reduction, of interacting gauge and scalar fields. Classical properties of the model are very good: there are vacuum solutions which include the trivial vacuum, the BRST invariance is established [22]. The perturbative quantization was started in [23] with the calculation of divergences of the first order in the gauge coupling: the obtained divergences were IR logarithmic and included the tadpole. It was, however, hard to systematize the computation of the prefactors. The 2-point divergences which were found can be removed by the usual mass and wave-function renormalizations, but the tadpole diagram remains, signaling instability of the trivial vacuum under quantum fluctuations.

We continue here investigation of the quantization properties of the proposed gauge model. We calculate one-loop corrections to the propagators of second order; we find a systematic way to compute divergent integrals with two or more parameter integrations, which enables us to compare and add various contributions. However, in addition to the local terms, we find new 'nonlocal' infrared divergences of the $\square^{-1}$ and the $\square^{-2}$ type. Such terms do not exist in the classical action, thus rendering the theory nonrenormalizable.

The paper is organized as follows. In Sect. 2 we define and briefly review properties of the truncated Heisenberg space and the Yang-Mills theory on it, recollecting results from [22]. In Sect. 3 we go through the main steps and some details of the calculation and list additional propagator corrections, completing the earlier result [23]. In Sect. 4 we discuss the meaning of the obtained results and possibilities to improve the model. Important details of calculation are given in the appendices.

\section{Fields on the truncated Heisenberg space}

Truncated Heisenberg space is a noncommutative space $\mathcal{A}$ generated by three hermitian coordinates $x, y, z$ which satisfy the commutation relations

$$
\begin{aligned}
& {[x, y]=i \epsilon \mu^{-2}(1-\mu z), \quad[x, z]=i \epsilon(y z+z y),} \\
& {[y, z]=-i \epsilon(x z+z x) .}
\end{aligned}
$$

The constant $\mu$ has dimension of the inverse length and $\epsilon$ is a dimensionless noncommutativity parameter. For $\epsilon=1$ algebra (2.1) has finite-dimensional matrix representations; $\epsilon=0$ defines the 'commutative limit'. Double scaling limit $\mu \rightarrow 0, \epsilon \rightarrow 0, k=\epsilon \mu^{-2}=$ finite reduces (2.1) to the Heisenberg algebra

$[x, y]=i k$.

The irreducible representation of the Heisenberg algebra is infinite-dimensional; in the geometric context it is called the Moyal plane. Truncation of infinite matrices $x, y, z$ given in the Fock representation to finite $n \times n$ matrices gives algebra (2.1). In this sense the Heisenberg algebra (2.2) is a contraction, or $z=0$ subspace, of the truncated Heisenberg space [24]. The limit $n \rightarrow \infty$, which transforms (2.1) to (2.2), is a weak operator limit.

The truncated Heisenberg algebra can be endowed with a differential structure. The space of 1-forms is spanned by frame $\left\{\theta^{\alpha}\right\}, \alpha=1,2,3$; derivations $e_{\beta}$ dual to $\theta^{\alpha}$ are defined to satisfy $\theta^{\alpha}\left(e_{\beta}\right)=\delta_{\beta}^{\alpha}$. We assume [25] 
$\left[f, \theta^{\alpha}\right]=0, \quad \mathrm{~d} f=\left(e_{\alpha} f\right) \theta^{\alpha}=\left[p_{\alpha}, f\right] \theta^{\alpha}$.

The frame derivations $e_{\alpha}$ are inner and generated by momenta $p_{\alpha} \in \mathcal{A} ; p_{\alpha}$ are, by convention, antihermitian. An important property of the inner-derivation calculus is the existence of a special connection

$\theta=-p_{\alpha} \theta^{\alpha}$

which generates the differential, $\mathrm{d} f=-[\theta, f]$. We choose

$\epsilon p_{1}=i \mu^{2} y, \quad \epsilon p_{2}=-i \mu^{2} x, \quad \epsilon p_{3}=i \mu\left(\mu z-\frac{1}{2}\right)$.

It can easily be seen that for $z=0$ this differential reduces to the standard one on the Moyal plane.

The algebra of momenta is in general quadratic [25]

$2 P^{\gamma \delta}{ }_{\alpha \beta} p_{\gamma} p_{\delta}-F_{\alpha \beta}^{\gamma} p_{\gamma}-\frac{1}{i \epsilon} K_{\alpha \beta}=0$,

the $K_{\alpha \beta}, F^{\gamma}{ }_{\alpha \beta}$, and $P^{\gamma \delta}{ }_{\alpha \beta}$ are constants. It defines a noncommutative wedge product. The Hodge dual on the other hand cannot be defined in the general case as it depends on (the existence of) the trace: in our case it is almost unique [21]. Finally, one specifies the connection: the metric-compatible connection used in [20] defines a noncommutative space with curvature and torsion.

The $U(1)$ gauge symmetry is introduced through the gauge potential $A$, which is an antihermitian 1-form, and the field strength $F$ :

$\mathrm{A}=i \mathrm{gA}_{\alpha} \theta^{\alpha}, \quad \mathrm{F}=\mathrm{dA}+\mathrm{A}^{2}=\frac{i}{2} \mathrm{~F}_{\alpha \beta} \theta^{\alpha} \theta^{\beta}$.

The $g$ denotes the $U$ (1) coupling constant; the $U(1)$ group consists of all unitary elements of $\mathcal{A}$. A remarkable property of noncommutative differential which we use is a possibility to construct a gauge-covariant 1-form: the difference

$\mathbf{X}=\mathrm{X}_{\alpha} \theta^{\alpha}=\mathrm{A}-\theta, \quad \mathbf{X}_{\alpha}=p_{\alpha}+i \mathrm{gA}_{\alpha}$

transforms in the adjoint representation of the gauge group. The coefficients $\mathrm{X}_{\alpha}$ are called covariant coordinates (a more appropriate name would perhaps be covariant momenta). Expressing the field strength in terms of $\mathrm{X}$ and the structure constants we find

$$
\mathbf{F}=\mathbf{X}^{2}-\frac{1}{2} F_{\alpha \beta}^{\gamma} \mathbf{X}_{\gamma} \theta^{\alpha} \theta^{\beta}-\frac{1}{2 i \epsilon} K_{\alpha \beta} \theta^{\alpha} \theta^{\beta} .
$$

The existence of $\mathrm{X}$ means that there are covariant observables which depend only on the potentials, and it opens a possibility to define alternative actions for gauge fields, with different properties from the Yang-Mills or the Chern-Simons actions. This is a new effect characteristic for noncommutative spaces. Our model is, however, built as a noncommutative generalization of the Yang-Mills theory so we shall keep only the original YM term in the action; we discuss possible new terms in the last section.

The YM action on the truncated Heisenberg space is given by

$\mathcal{S}_{Y M}=\frac{1}{16 \mathrm{~g}^{2}} \operatorname{Tr}(\mathrm{F}(* \mathrm{~F})+(* \mathrm{~F}) \mathrm{F})$.

Dimensional reduction to $z=0$ is done by considering only fields $\mathrm{A}_{\alpha}(x, y, z=0$ ), by (formally) integrating over $z$, and by rescaling the gauge coupling constant $(g \rightarrow g)$ and gauge fields. This gives the Kaluza-Klein reduced action on the Moyal plane. In order to distinguish the values of the gauge fields $\mathrm{A}_{\alpha}, \mathrm{F}_{\alpha \beta}, \alpha, \beta=1,2,3$, defined in three dimensions from the gauge fields defined intrinsically on the Moyal plane, we denote the latter by $A_{\alpha}, F_{\alpha \beta}, \alpha, \beta=1,2$. Fields and coupling constants have different mass dimension in two and three dimensions: dimensional reduction procedure takes care of this automatically. For $z=0$ the third component of the momentum is constant, $p_{3}=-i \mu / 2 \epsilon, e_{3}=0$, and $\mathrm{A}_{3}$ transforms as a scalar field in the adjoint representation. We denote

$\mathrm{gA}_{3}=g \phi, \quad \mathrm{gA}_{1}=g A_{1}, \quad \mathrm{gA}_{2}=g A_{2}$.

The field strength and covariant derivative in two dimensions are defined as

$$
\begin{aligned}
& D_{\alpha} \phi=e_{\alpha} \phi+i g\left[A_{\alpha}, \phi\right], \\
& \quad g^{-1} F_{12}=e_{1} A_{2}-e_{2} A_{1}+i g\left[A_{1}, A_{2}\right] .
\end{aligned}
$$

After the KK reduction, components of the three-dimensional $\mathrm{F}$ become [22]

$g^{-1} \mathrm{~F}_{12}=g^{-1} F_{12}-\mu \phi=g^{-1}\left(-i\left[\mathrm{X}_{1}, \mathrm{X}_{2}\right]+\frac{\mu^{2}}{\epsilon}\right)-\mu \phi$,

$\mathrm{g}^{-1} \mathrm{~F}_{13}=D_{1} \phi-i \epsilon\left\{p_{2}+i g A_{2}, \phi\right\}=\left[\mathrm{X}_{1}, \phi\right]-i \epsilon\left\{\mathrm{X}_{2}, \phi\right\}$,

$\mathrm{g}^{-1} \mathrm{~F}_{23}=D_{2} \phi+i \epsilon\left\{p_{1}+i g A_{1}, \phi\right\}=\left[\mathrm{X}_{2}, \phi\right]+i \epsilon\left\{\mathrm{X}_{1}, \phi\right\}$.

Introducing

$a=1-\epsilon^{2}$

we obtain

$\mathcal{S}_{Y M}=\frac{1}{2 \mathrm{~g}^{2}} \operatorname{Tr}\left(a \mathrm{~F}_{12} \mathrm{~F}^{12}+\mathrm{F}_{13} \mathrm{~F}^{13}+\mathrm{F}_{23} \mathrm{~F}^{23}\right)$,

that is,

$$
\begin{aligned}
\mathcal{S}_{Y M}= & \frac{1}{2} \operatorname{Tr}\left(\frac{a}{g^{2}}\left(F_{12}\right)^{2}-\frac{2 a \mu}{g} F_{12} \phi\right. \\
& +(4+a) \mu^{2} \phi^{2}-4 \epsilon F_{12} \phi^{2} \\
& +\left(D_{1} \phi\right)^{2}+\left(D_{2} \phi\right)^{2}-\epsilon^{2}\left\{p_{1}+i g A_{1}, \phi\right\}^{2}
\end{aligned}
$$




$$
\begin{aligned}
& \left.-\epsilon^{2}\left\{p_{2}+i g A_{2}, \phi\right\}^{2}\right) \\
= & \frac{1}{2} \operatorname{Tr}\left(-\frac{a}{g^{2}}\left[\mathrm{X}_{1}, \mathrm{X}_{2}\right]^{2}+a \mu^{2} \phi^{2}\right. \\
& -\frac{2 a \mu^{3}}{g \epsilon} \phi+\frac{2 i a \mu}{g}\left[\mathrm{X}_{1}, \mathrm{X}_{2}\right] \phi \\
& +4 i \epsilon\left[\mathrm{X}_{1}, \mathrm{X}_{2}\right] \phi^{2}+\left[\mathrm{X}_{1}, \phi\right]^{2} \\
& \left.+\left[\mathrm{X}_{2}, \phi\right]^{2}-\epsilon^{2}\left\{\mathrm{X}_{1}, \phi\right\}^{2}-\epsilon^{2}\left\{\mathrm{X}_{2}, \phi\right\}^{2}\right) .
\end{aligned}
$$

The two expressions are the same up to terms which are constant or proportional to a commutator, that is, to surface and cosmological constant terms. ${ }^{1}$

Let us briefly analyze the actions (2.9) and (2.15). Clearly, they are defined only when the trace is defined, that is, in a fixed representation of the algebra. One way to proceed is to consider finite matrix representations, that is, $\epsilon=1$, $a=0$ : it gives a non-propagating gauge field which interacts with the scalar. Another possibility, which we choose here, is to go to the continuous limit and represent fields on the Moyal space. There are various advantages and drawbacks of this choice. On the one hand, the resulting action is relatively complicated as gauge and scalar fields are mixed in the kinetic term. This fact on the other hand indicates that the harmonic potential confines both fields, gauge and scalar. The action is manifestly gauge invariant, but the status of the LS duality is not clear: (2.15) is not invariant under the exchange $\left[\mathrm{X}_{1}, \mathrm{X}_{2}\right] \leftrightarrow\left\{\mathrm{X}_{1}, \mathrm{X}_{2}\right\}$. However, one hopes that the geometric origin of the action could induce cancelation of divergences as in supersymmetry.

The action (2.15) has two classical vacua,

$A_{1}=0, \quad A_{2}=0, \quad \phi=0$,

$A_{1}=-\frac{\mu^{2} y}{g \epsilon}, \quad A_{2}=\frac{\mu^{2} x}{g \epsilon}, \quad \phi=\frac{\mu}{g \epsilon}$.

The first is the usual trivial vacuum; the second describes a configuration with constant value of the field strength $F_{12}=$ $\mu^{2} / \epsilon$. In quantization we expand around the trivial vacuum. After gauge fixing and inclusion of the ghost terms, we obtain [23]

$S=S_{Y M}+S_{g f}+S_{g h}=S_{\mathrm{kin}}+S_{\mathrm{int}}$,

with

$$
\begin{aligned}
S_{\text {kin }}= & -\frac{1}{2} \int a A_{\alpha} \square A^{\alpha}+2 a \mu \epsilon^{\alpha \beta}\left(\partial_{a} A_{\beta}\right) \phi+\phi \square \phi \\
& -(4+a) \mu^{2} \phi^{2}-4 \mu^{4} x^{\alpha} x_{\alpha} \phi^{2}+2 \bar{c} \square c,
\end{aligned}
$$

The background noncommutative space is curved but gravity is not dynamical.

$$
\begin{aligned}
& S_{\text {int }}=-\frac{1}{2} \int 4 \epsilon g \epsilon_{\alpha \beta}\left(\partial^{\alpha} A^{\beta}+i g A^{\alpha} \star A^{\beta}\right) \star \phi^{2} \\
& -2 i g\left(\partial_{\alpha} \phi\right)\left[A^{\alpha} \stackrel{\star}{,} \phi\right] \\
& +2 i a \mu g \epsilon_{\alpha \beta} A^{\alpha} \star A^{\beta} \phi-2 i a g \epsilon_{\alpha \beta} \partial^{\alpha} A^{\beta} \epsilon_{\gamma \beta} A^{\gamma} \star A^{\delta} \\
& +a g^{2}\left(\epsilon_{\alpha \beta} A^{\alpha} \star A^{\beta}\right)^{2}+g^{2}\left[A_{\alpha} \stackrel{\star}{,} \phi\right]\left[A^{\alpha}, \phi\right] \\
& -\epsilon^{2} g^{2}\left\{A_{\alpha} \stackrel{\star}{,} \phi\right\}\left\{A^{\alpha} \stackrel{\star}{,} \phi\right\} \\
& +2 \mu^{2} \epsilon g \epsilon_{\alpha \beta}\left\{x^{\alpha}, \phi\right\}\left\{A^{\beta}, \phi\right\}-i g \bar{c} \partial_{\alpha}\left[A^{\alpha}, c\right] \text {. }
\end{aligned}
$$

This is the action which we will analyze.

\section{Propagators: the one-loop structure}

Let us recall some results from [23] and introduce new notation which enables us to perform calculations more efficiently. We start with the kinetic term. The scalar and gauge fields in the kinetic term are mixed: because of noncommutativity it is not possible to diagonalize it. We therefore consider fields as multiplet $\Phi^{T}=\left(A^{\mu}, \phi\right)$ (which they were before the KK reduction), and write the kinetic term as

$$
\begin{aligned}
S_{\text {kin }}= & -\frac{1}{2} \int\left(A^{\mu} \phi\right)\left(\begin{array}{ll}
a \square \delta_{\mu \nu} & -a \mu \epsilon_{\mu \xi} \partial^{\xi} \\
a \mu \epsilon_{\nu \eta} \partial^{\eta} & K^{-1}-a \mu^{2}
\end{array}\right)\left(\begin{array}{l}
A^{\nu} \\
\phi
\end{array}\right) \\
& +2 \bar{c} \square c \\
= & -\frac{1}{2} \int \Phi^{T} G^{-1} \Phi+2 \bar{c} \square c
\end{aligned}
$$

where we introduced

$K^{-1}=\square-4 \mu^{4} x_{\alpha} x^{\alpha}-4 \mu^{2}$.

The corresponding inverse operator, the momentum-space Mehler kernel for the massive scalar field, has in two dimensions the following parametric form [26]:

$K(r, s)=-\frac{\pi}{4 \mu^{4}} \int_{1}^{\infty} \frac{\mathrm{d} \xi}{\xi} \frac{\xi-1}{\xi+1} e^{-\frac{1}{8 \mu^{2}}\left((r+s)^{2} \xi+(r-s)^{2} \frac{1}{\xi}\right)}$.

Mass of the scalar field is $2 \mu$; for other values of mass, factor $(\xi-1) /(\xi+1)$ has a different exponent. We denote

$\tilde{r}^{\mu}=\epsilon^{\mu v} r_{\nu}, \quad r \wedge s=\frac{\epsilon}{\mu^{2}} \epsilon_{\mu \nu} r^{\mu} s^{\nu}=\frac{\epsilon}{\mu^{2}} r \cdot \tilde{s}$.

The momentum-space kernel of the kinetic operator is

$$
\begin{aligned}
& G^{-1}(r, s) \\
& \quad=\left(\begin{array}{ll}
-a r^{2} \delta_{\mu \nu}(2 \pi)^{2} \delta(r+s) & -i a \mu \tilde{r}_{\mu}(2 \pi)^{2} \delta(r+s) \\
-i a \mu \tilde{s}_{\nu}(2 \pi)^{2} \delta(r+s) & -a \mu^{2}(2 \pi)^{2} \delta(r+s)+K^{-1}(r, s)
\end{array}\right) .
\end{aligned}
$$

Inverting it, for the matrix elements of the propagator $G(r, s)$ we obtain 
$\phi(r) \phi(s)=K(r, s)$

$A^{\alpha}(r) \phi(s)=-i \mu \frac{\tilde{r}^{\alpha}}{r^{2}} K(r, s)$,

$\underbrace{\alpha}(r) A^{\beta}(s)=(-i \mu)^{2} \frac{\tilde{r}^{\alpha} \tilde{s}^{\beta}}{r^{2} s^{2}} K(r, s)-\frac{(2 \pi)^{2}}{a} \frac{\delta^{\alpha \beta} \delta(r+s)}{r^{2}}$.

These matrix elements obey the recurrence relations

$$
\begin{aligned}
& A^{\alpha}(r) \phi(s)=-i \mu \frac{\tilde{r}^{\alpha}}{r^{2}} \phi(r) \phi(s), \\
& A^{\alpha}(r) A^{\beta}(s)=(-i \mu)^{2} \frac{\tilde{r}^{\alpha} \tilde{s}^{\beta}}{r^{2} s^{2}} \phi(r) \phi(s)-\frac{(2 \pi)^{2}}{a} \frac{\delta^{\alpha \beta} \delta(r+s)}{r^{2}},
\end{aligned}
$$

which we will later use.

The interaction contains three- and four-vertices. In momentum space they are

$$
\begin{aligned}
& S_{\text {int }, 1}=-\frac{2 i \epsilon g}{(2 \pi)^{4}} \int \mathrm{d} p \mathrm{~d} q \mathrm{~d} k \delta(p+q+k) \\
& \times \cos \frac{p \wedge q}{2} \tilde{p}^{\mu} A_{\mu}(p) \phi(q) \phi(k) \\
& S_{i n t, 2}=\frac{2 i g}{(2 \pi)^{4}} \int \mathrm{d} p \mathrm{~d} q \mathrm{~d} k \delta(p+q+k) \\
& \times \sin \frac{p \wedge q}{2} p^{\mu} \phi(p) \phi(q) A_{\mu}(k), \\
& S_{\text {int }, 3}=-\frac{4 i \epsilon \mu^{2} g}{(2 \pi)^{4}} \int \mathrm{d} p \mathrm{~d} q \mathrm{~d} k \delta(p+q+k) \\
& \times \cos \frac{p \wedge q}{2} \frac{\partial}{\partial \tilde{p}_{\mu}} \phi(p) \phi(q) A_{\mu}(k), \\
& S_{\text {int }, 4}=-\frac{a \mu g}{(2 \pi)^{4}} \int \mathrm{d} p \mathrm{~d} q \mathrm{~d} k \delta(p+q+k) \\
& \times \sin \frac{p \wedge q}{2} \epsilon^{\mu \nu} A_{\mu}(p) A_{\nu}(q) \phi(k), \\
& S_{\text {int }, 5}=\frac{i a g}{(2 \pi)^{4}} \int \mathrm{d} p \mathrm{~d} q \mathrm{~d} k \delta(p+q+k) \\
& \times \sin \frac{p \wedge q}{2} \epsilon^{\mu \nu} \tilde{k}^{\rho} A_{\mu}(p) A_{v}(q) A_{\rho}(k), \\
& S_{\text {int }, 6}=\frac{2 i g}{(2 \pi)^{4}} \int \mathrm{d} p \mathrm{~d} q \mathrm{~d} k \delta(p+q+k) \\
& \times \sin \frac{p \wedge q}{2} p^{\mu} \bar{c}(p) c(q) A_{\mu}(k), \\
& S_{\text {int }, 7}=\frac{2 g^{2}}{(2 \pi)^{6}} \int \mathrm{d} p \mathrm{~d} q \mathrm{~d} p^{\prime} \mathrm{d} q^{\prime} \delta\left(p+q+p^{\prime}+q^{\prime}\right) \\
& \times \sin \frac{p \wedge q}{2} \sin \frac{p^{\prime} \wedge q^{\prime}}{2} \\
& \times \delta^{\mu v} A_{\mu}(p) \phi(q) A_{v}\left(p^{\prime}\right) \phi\left(q^{\prime}\right), \\
& S_{\text {int }, 8}=\frac{2 \epsilon^{2} g^{2}}{(2 \pi)^{6}} \int \mathrm{d} p \mathrm{~d} q \mathrm{~d} p^{\prime} \mathrm{d} q^{\prime} \delta\left(p+q+p^{\prime}+q^{\prime}\right) \\
& \times \cos \frac{p \wedge q}{2} \cos \frac{p^{\prime} \wedge q^{\prime}}{2}
\end{aligned}
$$

$$
\begin{aligned}
& \times \delta^{\mu \nu} A_{\mu}(p) \phi(q) A_{v}\left(p^{\prime}\right) \phi\left(q^{\prime}\right), \\
S_{i n t, 9}= & -\frac{2 \epsilon g^{2}}{(2 \pi)^{6}} \int \mathrm{d} p \mathrm{~d} q \mathrm{~d} p^{\prime} \mathrm{d} q^{\prime} \mathrm{d}\left(p+q+p^{\prime}+q^{\prime}\right) \\
& \times \sin \frac{p \wedge q}{2} \cos \frac{p^{\prime} \wedge q^{\prime}}{2} \\
& \times \epsilon^{\mu \nu} A_{\mu}(p) A_{v}(q) \phi\left(p^{\prime}\right) \phi\left(q^{\prime}\right), \\
S_{i n t, 10}= & \frac{a g^{2}}{2(2 \pi)^{6}} \int \mathrm{d} p \mathrm{~d} q \mathrm{~d} p^{\prime} \mathrm{d} q^{\prime} \mathrm{d}\left(p+q+p^{\prime}+q^{\prime}\right) \\
& \times \sin \frac{p \wedge q}{2} \sin \frac{p^{\prime} \wedge q^{\prime}}{2} \epsilon^{\mu \nu} \\
& \times \epsilon^{\rho \sigma} A_{\mu}(p) A_{v}(q) A_{\rho}\left(p^{\prime}\right) A_{\sigma}\left(q^{\prime}\right) .
\end{aligned}
$$

We want to calculate the one-loop corrections to the propagators, that is, the sum of the expectation values,

$$
P_{F F^{\prime}, i j}(r, s)=-\left\langle F(r) F^{\prime}(s) S_{\mathrm{int}, i} S_{\mathrm{int}, j}\right\rangle, \quad i, j=1, \ldots, 6,
$$

$$
P_{F F^{\prime}, i}(r, s)=-\left\langle F(r) F^{\prime}(s) S_{\mathrm{int}, i}\right\rangle, \quad i=7, \ldots, 10, \text { (3.9) }
$$

where $F$ an $F^{\prime}$ are fields $\phi$ or $A^{\mu}$ and $i, j$ label different interaction vertices, 1-10. Expressions of the form (3.8) correspond to the 2-point functions; (3.9) are the 1-point functions. We have previously calculated divergent 1-point functions $P_{F, i}$ [23]. In fact, as we wish to obtain divergent terms as they appear in the effective action, we can go a step further and calculate the amputated graphs $\Pi(r, s)$. The removal of the external legs of $P(r, s)$ is nontrivial because of the Mehler propagators and amounts to

$\Pi(p, q)=\frac{1}{(2 \pi)^{4}} \int \mathrm{d} r \mathrm{~d} s G^{-1}(p,-r) P(r, s) G^{-1}(-s, q)$.

In fact, it simplifies the final result as it decreases the number of the Mehler-kernel factors, that is, the number of parameter integrals.

Due to the recurrence relations (3.7) all field contraction reduce to contractions of the scalar fields. Let us introduce shorthand notation for multiple contractions. In the case of two Mehler kernels we denote

$$
\begin{aligned}
K_{2}(r, s, p, q)= & K(r, s) K(p, q)+K(r, p) K(s, q) \\
& +K(r, q) K(s, p) .
\end{aligned}
$$

When there are several external momenta (in this case $r$ and $s$ ), we separate them from the internal ones by a vertical line and write

$$
K_{2}(r, s \mid p, q)=K(r, p) K(s, q)+K(r, q) K(s, p) .
$$

With $m$ external and $n$ internal momenta $(n \geq m)$, this generalizes to $K_{m+n}$, defined as

$$
2^{\frac{n-m}{2}}\left(\frac{n-m}{2}\right) ! K_{m+n}\left(r_{1}, \ldots, r_{m} \mid p_{1}, \ldots, p_{n}\right)
$$




$$
\begin{aligned}
= & \sum_{\pi_{p}} K\left(r_{1}, p_{\pi_{1}}\right) K\left(r_{2}, p_{\pi_{2}}\right) \ldots K\left(r_{m}, p_{\pi_{m}}\right) \\
& \times K\left(p_{\pi_{m+1}}, p_{\pi_{m+2}}\right) \ldots K\left(p_{\pi_{n-1}}, p_{\pi_{n}}\right)
\end{aligned}
$$

where $\pi_{p}$ are permutations of the internal momenta. $K_{m+n}$ is symmetric under exchange of any two internal or any two external momenta. Calculation of contractions can also be aided by the recurrence relation

$$
\begin{aligned}
& K_{m+n}\left(r_{1}, \ldots, r_{m} \mid p_{1}, \ldots, p_{n}\right) \\
& =\sum_{i=1}^{n} K\left(r_{1}, p_{i}\right) K_{m+n-1} \\
& \quad \times\left(r_{2}, \ldots, r_{m} \mid p_{1}, \ldots, p_{i-1}, p_{i+1}, \ldots, p_{n}\right) .
\end{aligned}
$$

Applying (3.7), the sum of one-loop contributions can be simplified to

$$
\begin{aligned}
P_{\phi \phi} & =\sum_{i \leq j \leq 6}\left(2-\delta_{i j}\right) P_{\phi \phi, i j}+\sum_{7 \leq k \leq 10} P_{\phi \phi, k} \\
P_{\phi A}^{\alpha} & =\sum_{i \leq j \leq 6}\left(2-\delta_{i j}\right) P_{\phi A, i j}^{\alpha}+\sum_{7 \leq k \leq 10} P_{\phi A, k}^{\alpha} \\
& =-i \mu \frac{\tilde{r}^{\alpha}}{r^{2}} P_{\phi \phi}+P_{\phi A}^{\prime \alpha}, \\
P_{A A}^{\alpha \beta} & =\sum_{i \leq j \leq 6}\left(2-\delta_{i j}\right) P_{A A, i j}^{\alpha \beta}+\sum_{7 \leq k \leq 10} P_{A A, k}^{\alpha \beta} \\
& =-\mu^{2} \frac{\tilde{r}^{\alpha} \tilde{s}^{\beta}}{r^{2} s^{2}} P_{\phi \phi}-i \mu \frac{\tilde{r}^{\alpha}}{r^{2}} P_{\phi A}^{\prime \beta}-i \mu \frac{\tilde{s}^{\beta}}{s^{2}} P_{\phi A}^{\prime \alpha}+P_{A A}^{\prime \alpha \beta},
\end{aligned}
$$

where due to the similarity of vertices 4 and 5 we have

$$
\begin{aligned}
P_{\phi \phi, i 5}= & -P_{\phi \phi, i 4}+P_{\phi \phi, i 5}^{\prime}, \\
P_{\phi A, i 5}^{\alpha}= & -P_{\phi A, i 4}^{\alpha}-i \mu \frac{\tilde{r}^{\alpha}}{r^{2}} P_{\phi \phi, i 5}^{\prime}+P_{\phi A, i 5}^{\prime \alpha}, \\
P_{A A, i 5}^{\alpha \beta}= & -P_{A A, i 4}^{\alpha \beta}-\mu^{2} \frac{\tilde{r}^{\alpha} \tilde{s}^{\beta}}{r^{2} s^{2}} P_{\phi \phi, i 5}^{\prime}-i \mu \frac{\tilde{r}^{\alpha}}{r^{2}} P_{\phi A, i 5}^{\prime \beta} \\
& -i \mu \frac{\tilde{s}^{\beta}}{s^{2}} P_{\phi A, i 5}^{\prime \alpha},
\end{aligned}
$$

with $i=1, \ldots, 5$. This leads to a significant cancelation and absorption of terms.

In principle we have two kinds of divergent one-loop contributions to the propagators. The four-vertices give firstorder divergences which were found in [23]:

$$
\int \phi \phi, \int A^{\mu} A_{\mu}, \quad \int \epsilon^{\mu v} x_{\mu} A_{\nu} \phi .
$$

There are also second-order contributions from the threevertices which we calculate here. It is clear that, having so many types of interactions, there will be a large number of terms. We shall therefore not attempt to present our calculation to its full extent, but we will rather explain its logic and go through the main steps. Some parts of the calculation are straightforward albeit long; but to extract and quantify the final results we have to define a specific prescription adjusted to divergent multiple parameter integrals of rational expressions.

Let us first consider $P_{\phi \phi}$, which is the most divergent of the matrix elements. The diagram containing two vertices 1 is given by

$$
\begin{aligned}
P_{\phi \phi, 11}= & -\frac{4 \epsilon^{2} \mu^{2} g^{2}}{(2 \pi)^{8}} \int \mathrm{d} p \mathrm{~d} q \mathrm{~d} k \mathrm{~d} p^{\prime} \mathrm{d} q^{\prime} \mathrm{d} k^{\prime} \\
& \times \delta(p+q+k) \delta\left(p^{\prime}+q^{\prime}+k^{\prime}\right) \cos \frac{p \wedge q}{2} \cos \frac{p^{\prime} \wedge q^{\prime}}{2} \\
& \times \epsilon^{\rho \sigma} p_{\rho} \epsilon^{\mu v} p_{\mu}^{\prime}\left\langle\phi(r) \phi(s) A_{\sigma}(p) \phi(q) \phi(k)\right. \\
& \left.\times A_{\nu}\left(p^{\prime}\right) \phi\left(q^{\prime}\right) \phi\left(k^{\prime}\right)\right\rangle,
\end{aligned}
$$

where the correlation function $\left\langle\phi(r) \phi(s) A_{\sigma}(p) \phi(q) \phi(k)\right.$ $\left.A_{v}\left(p^{\prime}\right) \phi\left(q^{\prime}\right) \phi\left(k^{\prime}\right)\right\rangle$ is a sum of contractions of external fields with fields in the vertices. There are 90 terms of the type

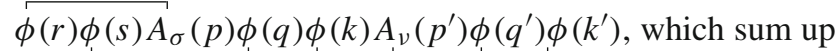
to $K_{4}\left(r, s \mid p, q, k, p^{\prime}, q^{\prime}, k^{\prime}\right)$ and 12 terms with the $A A$ contractions which produce $K_{3}\left(r, s \mid p, q, p^{\prime}, q^{\prime}\right): K_{3}$ and $K_{4}$ play the role of the usual symmetry factors. We find

$$
\begin{aligned}
P_{\phi \phi, 11}= & -\frac{4 \epsilon^{2} \mu^{2} g^{2}}{(2 \pi)^{8}} \int \mathrm{d} p \mathrm{~d} q \mathrm{~d} k \mathrm{~d} p^{\prime} \mathrm{d} q^{\prime} \mathrm{d} k^{\prime} \\
& \times \delta(p+q+k) \delta\left(p^{\prime}+q^{\prime}+k^{\prime}\right) \\
& \times \cos \frac{p \wedge q}{2} \cos \frac{p^{\prime} \wedge q^{\prime}}{2} K_{4}\left(r, s \mid p, q, k, p^{\prime}, q^{\prime}, k^{\prime}\right) \\
& +\frac{4 \epsilon^{2} g^{2}}{(2 \pi)^{6} a} \int \mathrm{d} p \mathrm{~d} q \mathrm{~d} p^{\prime} \mathrm{d} q^{\prime} \delta\left(p+q+p^{\prime}+q^{\prime}\right) \\
& \times \cos \frac{p \wedge q}{2} \cos \frac{p^{\prime} \wedge q^{\prime}}{2} K_{3}\left(r, s \mid p, q, p^{\prime}, q^{\prime}\right) .
\end{aligned}
$$

Contributions of other vertices to the $P_{\phi \phi}$ propagator are given in Appendix 1. Representing the $\phi \phi$ propagator by a straight line, the $A A$ by a wiggly line and the $\phi A$ by a mixed line, these contributions correspond to diagrams

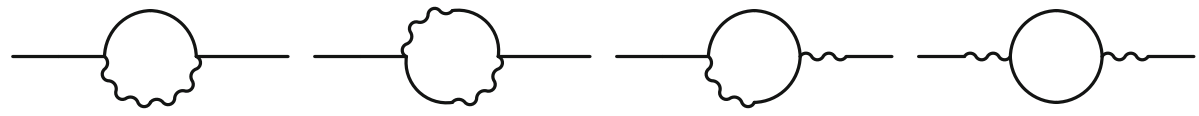


The one-loop quantum correction is the sum of all enumerated terms. In order to calculate it one first expresses Mehler kernels $K_{3}, K_{4}$ etc. as parameter integrals which are Gaussian in $p, q, k$; momentum integration can then be performed. ${ }^{2}$ The result is a divergent multiple parameter integral which, except in the simplest cases, cannot be done explicitly when there is more than one integration.

A more useful way to analyze divergences is to use the amputated propagators, in which $K_{3}$ and $K_{4}$ for example reduce to $K$ and $K_{2}$. But in order to find the amputated propagators we need to find all matrix elements, that is, $P_{\phi A}$ and $P_{A A}$ as well. They are of a form similar to $P_{\phi \phi}$ and somewhat longer; the full expressions are given in [27]. Multiplication of the amputated propagator by a multiplet of classical external fields gives the one-loop effective action:

$\Gamma=\frac{1}{2} \int \mathrm{d} r \mathrm{~d} s \Phi^{T}(-r) \Pi(r, s) \Phi(-s)$.

Our main goal is to extract the divergent parts of the last expression. We find

$$
\begin{aligned}
\Pi^{\mu v}(p, q)= & a^{2} p^{2} q^{2} P_{A A}^{\prime \mu \nu}(p, q) \\
\Pi^{\mu}(p, q)= & -i a^{2} \mu p^{2} \tilde{q}_{\rho} P_{A A}^{\prime \mu \rho}(p, q) \\
& -\frac{a p^{2}}{(2 \pi)^{2}} \int \mathrm{d} k P_{\phi A}^{\prime \mu}(p, k) K^{-1}(-k, q) \\
\Pi(p, q)= & -a^{2} \mu^{2} \tilde{p}_{\rho} \tilde{q}_{\sigma} P_{A A}^{\prime \rho \sigma}(p, q) \\
& +\frac{i a \mu \tilde{p}_{\rho}}{(2 \pi)^{2}} \int \mathrm{d} k P_{\phi A}^{\prime \rho}(p, k) K^{-1}(-k, q) \\
& +\frac{i a \mu \tilde{q}_{\rho}}{(2 \pi)^{2}} \int \mathrm{d} k P_{\phi A}^{\prime \rho}(q, k) K^{-1}(-k, p) \\
& +\frac{1}{(2 \pi)^{4}} \int \mathrm{d} p^{\prime} \mathrm{d} q^{\prime} K^{-1}\left(p,-p^{\prime}\right) P_{\phi \phi}\left(p^{\prime}, q^{\prime}\right) \\
& \times K^{-1}\left(-q^{\prime}, q\right) .
\end{aligned}
$$

In comparison to full propagators, these expressions are considerably simpler. For exact forms of $P_{\phi A}^{\prime \rho}$ and $P_{A A}^{\prime \rho \sigma}$ we refer to [28].

\section{Divergences}

\subsection{The $\phi \phi$-sector}

Part of the effective action which gives the one-loop quantum corrections to the propagators is given by (3.23). In the usual case, 2-point functions have the form

\footnotetext{
2 This is true if the denominator of the rational expression which appears as a factor in the course of integrations is of relatively low degree: otherwise one has to introduce additional Schwinger parametrizations.
}

$\Pi(r, s)=\Pi(r) \delta(r+s)$

which reflects the translational invariance. (In our convention for the Fourier transformation, all momenta are incoming.) However, we are dealing with a nonlocal action which is not translationally invariant. Therefore in order to recover the form of divergences in the effective action in position space we introduce the so-called 'short' and 'long variable', respectively, $u$ and $v$ :

$u=\frac{r+s}{2}, \quad v=\frac{r-s}{2}$.

Here $u$ denotes the difference between the incoming and outgoing momenta in a vertex or along a line. In translationally invariant case one integrates over $u$ and the divergences remain in $\Pi(v)$,

$$
\begin{aligned}
\Gamma & =\int \mathrm{d} u \mathrm{~d} v \Phi^{T}(-u-v) \Pi(u+v) \delta(2 u) \Phi(-u+v) \\
& =\frac{1}{2} \int \mathrm{d} v \Phi^{T}(-v) \Pi(v) \Phi(v) .
\end{aligned}
$$

Here the $\delta$-function is smeared, roughly replaced by an exponentially decreasing factor

$\delta(u)=\lim _{\sigma \rightarrow 0} \frac{1}{2 \pi \sigma^{2}} e^{-\frac{u^{2}}{2 \sigma^{2}}}$,

which is hidden in parameter integrations. The exponential factors regularize all momentum integrations in the UV sector: divergences occur in the IR sector, for small values of $u$. Our strategy to calculate them is as follows. We expand terms in the effective action (3.23) around $u=0$, keeping all parameter integrals which come from the Mehler kernels and Schwinger parametrizations. This gives momentum integrals of the Poisson type (which one can calculate) and usually leaves two parameter integrations. In order to identify the types of divergences we introduce appropriate regulators, expand fields in powers of $u_{\alpha}$ as in (4.5), and integrate term by term: only the first few terms are infinite. As mentioned, we consider only the lower bound in momentum integrals as that is where divergences lie. Eventually, we find new nonlocal divergences of the form

$\int \phi \square^{-1} \phi, \quad \int \phi \square^{-2} \phi$

Let us discuss details of the calculation of $\Gamma_{\phi \phi}^{(d i v)}$. After removal of the external legs we obtain lengthy expression which contains several hundred terms. Most of them are finite, which can be checked by power counting. We consider divergent terms in the increasing order of powers of the momentum, expecting to find in the lowest order only mass and wave-function renormalizations. However, a closer 
inspection shows that divergent terms of the lowest degree can, and do, contain nonlocal $\square^{-2}$ and $\square^{-1}$ terms which are new. We focus therefore on them; we denote the corresponding parts of $\Pi, \Gamma$ by a tilde. Parts of the amputated propagator $\Pi$ which contain nonlocal divergent contributions are

$$
\begin{aligned}
\tilde{\Pi}_{\phi \phi}^{(1)}= & -\frac{32 a \mu^{8} g^{2}}{(2 \pi)^{2} \epsilon^{2}} \frac{r \wedge s}{r^{2} s^{2}(r+s)^{2}} \sin \frac{r \wedge s}{2} \int \mathrm{d} p \mathrm{~d} q \\
& \times \delta(-r-s+p+q) \sin \frac{p \wedge q}{2} \frac{p \wedge q}{p^{2}} K(p, q), \\
\tilde{\Pi}_{\phi \phi}^{(2)}= & -\frac{8 a \mu^{8} g^{2}}{(2 \pi)^{2} \epsilon^{2}} \frac{1}{r^{2} s^{2}} \int \mathrm{d} p \mathrm{~d} q \delta(-r-s+p+q) \\
& \times \sin \frac{p \wedge r}{2} \sin \frac{q \wedge s}{2} \frac{(p \wedge r)(q \wedge s)}{p^{2} q^{2}} K(p, q), \\
\tilde{\Pi}_{\phi \phi}^{(3)}= & \frac{8 \mu^{4} g^{2}}{(2 \pi)^{2}} \frac{1}{r^{2} s^{2}} \int \mathrm{d} p \mathrm{~d} q \delta(-r-s+p+q) \\
& \times \sin \frac{p \wedge r}{2} \sin \frac{q \wedge s}{2} \frac{(p \cdot r)(q \cdot s)}{p^{2} q^{2}} K(p, q), \\
\tilde{\Pi}_{\phi \phi}^{(4)}= & -\frac{8 a \mu^{8} g^{2}}{(2 \pi)^{2} \epsilon^{2}} \frac{1}{r^{2} s^{2}} \int \mathrm{d} p \mathrm{~d} q \delta(-r-s+p+q) \\
& \times \sin \frac{p \wedge r}{2} \sin \frac{q \wedge s}{2} \frac{(p \wedge r)(q \wedge s)}{p^{2}(p-r)^{2}} K(p, q) \\
& +(r \leftrightarrow s) .
\end{aligned}
$$

Introducing the short and long variables and expressing the Mehler kernel in parametric form we find the following contributions to the effective action:

$$
\begin{aligned}
\tilde{\Gamma}_{\phi \phi}^{(1)}= & \frac{2 a g^{2}}{\pi} \Re \int \mathrm{d} u \mathrm{~d} v \frac{\phi(-u-v) \phi(-u+v)}{(u+v)^{2}(u-v)^{2} u^{2}} \\
& \times(v \cdot \tilde{u}) \tilde{u}_{\alpha} e^{-i u \wedge v} \int_{1}^{\infty} \frac{\mathrm{d} \xi \xi-1}{\xi} \frac{\xi}{\xi+1} e^{-\left(\xi+\frac{1}{\xi}\right) \frac{u^{2}}{2 \mu^{2}}} \\
& \times \int \mathrm{d} p p^{\alpha}\left(e^{-i \epsilon \frac{p \cdot \tilde{u}}{\mu^{2}}}-e^{i \epsilon \frac{p \cdot \tilde{u}}{\mu^{2}}}\right) e^{-\frac{1}{\xi} \frac{p^{2}}{2 \mu^{2}}+\frac{1}{\xi} \frac{p \cdot u}{\mu^{2}}}, \\
\tilde{\Gamma}_{\phi \phi}^{(2)}= & \frac{a g^{2}}{2 \pi \mu^{2}} \Re \int_{1}^{\infty} \mathrm{d} u \mathrm{~d} v \frac{\phi(-u-v) \phi(-u+v)}{(u+v)^{2}(u-v)^{2}} \\
& \times e^{i u \wedge v} \int_{1}^{\infty} \frac{\mathrm{d} \xi}{\xi} \frac{\xi-1}{\xi+1} \int_{0}^{\infty} \mathrm{d} \eta e^{-\left(\xi+\frac{1}{\xi}+4 \eta\right) \frac{u^{2}}{2 \mu^{2}}} \\
& \times \int^{\mathrm{d} p} \frac{(p \cdot(\tilde{u}+\tilde{v}))(p \cdot(\tilde{u}-\tilde{v})+2 u \cdot \tilde{v})}{p^{2}} \\
& \times e^{-\left(\frac{1}{\xi}+\eta\right) \frac{p^{2}}{2 \mu^{2}}+\left(\frac{1}{\xi}+2 \eta\right) \frac{p \cdot u}{\mu^{2}}}\left(e^{-i \epsilon \frac{p \cdot \tilde{v}}{\mu^{2}}}-e^{i \epsilon \frac{p \cdot \tilde{u}}{\mu^{2}}}\right), \\
\tilde{\Gamma}_{\phi \phi}^{(3)}= & \frac{g^{2}}{2 \pi \mu^{2}} \Re \int_{0}^{\infty} \mathrm{d} u \mathrm{~d} v \frac{\phi(-u-v) \phi(-u+v)}{(u+v)^{2}(u-v)^{2}} \\
& \times e^{i u \wedge v} \int_{1}^{\infty} \frac{\mathrm{d} \xi \xi}{\xi} \frac{\xi-1}{\xi+1} \int_{0}^{\infty} \mathrm{d} \eta e^{-\left(\xi+\frac{1}{\xi}+4 \eta\right) \frac{u^{2}}{2 \mu^{2}}}
\end{aligned}
$$

$$
\begin{aligned}
& \times \int \mathrm{d} p \frac{(p \cdot(u+v))((2 u-p) \cdot(u-v))}{p^{2}} \\
& \times e^{-\left(\frac{1}{\xi}+\eta\right) \frac{p^{2}}{2 \mu^{2}}+\left(\frac{1}{\xi}+2 \eta\right) \frac{p \cdot u}{\mu^{2}}}\left(e^{i \epsilon \frac{p \cdot \tilde{u}}{\mu^{2}}}-e^{-i \epsilon \frac{p \cdot \tilde{v}}{\mu^{2}}}\right), \\
\tilde{\Gamma}_{\phi \phi}^{(4)}= & \frac{a g^{2}}{2 \pi \mu^{2}} \Re \int \mathrm{d} u \mathrm{~d} v \frac{\phi(-u-v) \phi(-u+v)}{(u+v)^{2}(u-v)^{2}} \\
& \times e^{i u \wedge v} \int_{1}^{\infty} \frac{\mathrm{d} \xi}{\xi} \frac{\xi-1}{\xi+1} \int_{0}^{\infty} \mathrm{d} \eta \\
& \times e^{-\left(\xi+\frac{1}{\xi}+\eta\right) \frac{u^{2}}{2 \mu^{2}}-\eta \frac{v^{2}+2 u \cdot v}{2 \mu^{2}}} \\
& \times \int \mathrm{d} p \frac{(p \cdot(\tilde{u}+\tilde{v}))(p \cdot(\tilde{u}-\tilde{v})+2 u \cdot \tilde{v})}{p^{2}} \\
& \times e^{-\left(\frac{1}{\xi}+\eta\right) \frac{p^{2}}{2 \mu^{2}}+\left(\frac{1}{\xi}+\eta\right) \frac{p \cdot u}{\mu^{2}}+\eta \frac{p \cdot v}{\mu^{2}}}\left(e^{-i \epsilon \frac{p \cdot \tilde{v}}{\mu^{2}}}-e^{i \epsilon \frac{p \cdot \tilde{u}}{\mu^{2}}}\right) .
\end{aligned}
$$

In order to analyze the behavior of these integrals, we first perform the Gaussian integration over $p$. For $\tilde{\Gamma}_{\phi \phi}^{(1)}$, which is the simplest, we obtain

$$
\begin{aligned}
\Gamma_{\phi \phi}^{(1)}= & -2 a \epsilon g^{2} \int \mathrm{d} u \mathrm{~d} v \frac{\phi(-u-v) \phi(-u+v)}{(u+v)^{2}(u-v)^{2} u^{2}} \\
& \times(u \cdot \tilde{v}) \sin (u \wedge v) \int_{1}^{\infty} \mathrm{d} \xi \frac{\xi-1}{\xi+1} e^{-\left(1+\epsilon^{2}\right) \xi \frac{u^{2}}{2 \mu^{2}}} .
\end{aligned}
$$

We need to estimate this expression at the lower bound $u=0$, so we expand field $\phi$ around this point,

$\phi(-u+v)=\phi(v)-\partial_{\alpha} \phi(v) u^{\alpha}+\cdots$

The leading-order term is

$$
\begin{aligned}
\tilde{\Gamma}_{\phi \phi}^{(1)}= & -\frac{2 a \epsilon^{2} g^{2}}{\mu^{2}} \int \mathrm{d} v \frac{\phi(-v) \phi(v)}{v^{2}} \\
& \times \int \mathrm{d} u \int_{1}^{\infty} \mathrm{d} \xi \frac{\xi-1}{\xi+1} e^{-\left(1+\epsilon^{2}\right) \xi \frac{u^{2}}{2 \mu^{2}}} .
\end{aligned}
$$

One can easily see that this expression is divergent, that is, that the result of the last two integrations at the lower $u$-bound is infinite: we either put $u=0$, in which case the $\xi$-integral, $\int_{1}^{\infty} \mathrm{d} \xi(\xi-1) /(\xi+1)$, is divergent at $\xi=\infty$, or we first perform the $\xi$-integration and obtain $\int \mathrm{d} u e^{-u^{2}} / u^{2}$, which is logarithmically divergent at $u=0$.

Using the regularization described in Appendix 4, for the divergent part of $\tilde{\Gamma}_{\phi \phi}^{(1)}$ we obtain

$\tilde{\Gamma}_{\phi \phi}^{(1, d i v)}=-\frac{16 \pi^{3} a \epsilon^{2} g^{2}}{1+\epsilon^{2}} \log \Lambda \int \phi \square^{-1} \phi$,

where $\Lambda$ is the regularization parameter. The analysis of the remaining three terms is similar albeit more complicated, 
as the corresponding leading-order expressions, after expansion in $u$, contain integration in two parameters $\xi$ and $\eta$; relevant terms are written in Appendix 3. A systematic procedure which enables one to estimate these integrals, that is, to introduce a regulator and sum up different contributions, is described in Appendix 4. Adding all divergent nonlocal contributions in the $\phi \phi$-sector we obtain

$$
\begin{aligned}
\tilde{\Gamma}_{\phi \phi}^{(\text {div })}= & \left(\frac{8}{\epsilon^{2}}-14+\epsilon^{2}\right) \pi^{3} \mu^{4} g^{2} \log \Lambda \\
& \times \int \phi \square^{-2} \phi+\epsilon^{2} \pi^{3} \mu^{2} g^{2} \Lambda^{2} \int \phi \square^{-1} \phi .
\end{aligned}
$$

In addition, $\Gamma_{\phi \phi}^{(d i v)}$ contains the $\int \phi \phi$ term found before with a corrected infinite prefactor.

\subsection{The $A A$-sector}

The most important obstacle in constructing renormalizable noncommutative gauge theory on the Moyal space is quadratically divergent IR term of the form $\Pi^{\mu \nu} \propto p^{\mu} p^{v} /\left(p^{2}\right)^{2}$ which comes from the non-planar part of the gauge-field self energy [29-32] and seems to be independent on the gauge fixing. It gives rise to a nonlocal counterterm [33,34]

$$
\int F^{\mu \nu} \star \frac{1}{D^{2} \tilde{D}^{2}} \star F_{\mu \nu}
$$

As we will see, there is no such term in our theory, but other nonlocal terms exist.

Analyzing the form of $\Pi_{\mu v}$ we find only two amputatedpropagator terms in the $A A$ sector which can be sources of nonlocal divergences:

$$
\begin{aligned}
& \tilde{\Pi}_{\mu \nu}^{(1)}(r, s)=\frac{4 a \mu^{2} g^{2}}{(2 \pi)^{2}} \int \mathrm{d} p \mathrm{~d} q \delta(-r-s+p+q) \sin \frac{p \wedge r}{2} \\
& \times \sin \frac{q \wedge s}{2} \frac{p_{\mu} q_{\nu}}{p^{2} q^{2}} K(p, q) \\
& \quad \times \tilde{\Pi}_{\mu \nu}^{(2)}(r, s)=-\frac{8 a \mu^{2} g^{2}}{(2 \pi)^{2}} \int \mathrm{d} p \mathrm{~d} q \delta(-r-s+p+q) \\
& \quad \times \sin \frac{p \wedge r}{2} \sin \frac{q \wedge s}{2} \frac{\tilde{p}_{\mu} \tilde{q}_{\nu}}{p^{2} q^{2}} K(p, q) .
\end{aligned}
$$

In fact they are, up to replacement $p_{\mu} \rightarrow \tilde{p}_{\mu}, q_{\nu} \rightarrow \tilde{q}_{\nu}$, almost the same and they have the same divergent parts: we therefore analyze only the first. The computational details are very similar to those which we developed and explained in Appendix 4 for the $\phi \phi$-sector. As before, we want to examine the behavior of the integrals for small $u$. Introducing the short and long variables, $\tilde{\Pi}_{\mu \nu}^{(1)}(r, s)$ becomes

$$
\tilde{\Pi}_{\mu \nu}^{(1)}=\frac{a \mu^{2} g^{2}}{\pi^{2}} \int \mathrm{d} p \sin \frac{p \wedge(u+v)}{2}
$$

$$
\times \sin \frac{(2 u-p) \wedge(u-v)}{2} \frac{p_{\mu}(2 u-p)_{v}}{p^{2}(2 u-p)^{2}} K(p, 2 u-p) .
$$

Using the Schwinger parametrization and expressing the Mehler kernel in the parameter form, we obtain

$$
\begin{aligned}
& \tilde{\Pi}_{\mu \nu}^{(1)}(u, v)=-\frac{a}{8 \pi \mu^{4}} \int \mathrm{d} p(\cos (p \wedge u+u \wedge v) \\
& -\cos (p \wedge v-u \wedge v)) \frac{2 p_{\mu} u_{v}-p_{\mu} p_{v}}{p^{2}} \\
& \quad \times \int_{0}^{\infty} \mathrm{d} \eta e^{-\eta \frac{(2 u-p)^{2}}{2 \mu^{2}}} \int_{1}^{\infty} \frac{\mathrm{d} \xi}{\xi} \frac{\xi-1}{\xi+1} e^{-\frac{1}{2 \mu^{2}}\left(\xi u^{2}+\frac{1}{\xi}(p-u)^{2}\right)} .
\end{aligned}
$$

As we wish to single out terms proportional to $\tilde{v}^{\mu} \tilde{v}^{v} /\left(v^{2}\right)^{2}$ we can neglect the first cosine. After the Gaussian integration we obtain

$$
\begin{aligned}
\tilde{\Gamma}_{A A}^{(1)}= & -\frac{a g^{2}}{8 \mu^{2}} \Re \int_{1} \mathrm{~d} u \mathrm{~d} v A_{\mu}(-u-v) A_{v}(-u+v) \\
& \times e^{i u \wedge v} \int_{1}^{\infty} \mathrm{d} \xi \frac{\xi-1}{\xi+1} e^{-\xi \frac{u^{2}}{2 \mu^{2}}} \\
& \times \int_{0}^{\infty} \mathrm{d} \eta e^{-\frac{\eta}{1+\eta \xi} \frac{u^{2}}{2 \mu^{2}}} e^{-} \frac{\xi \epsilon^{2}}{1+\eta \xi} \frac{v^{2}}{2 \mu^{2}}+i \epsilon \frac{1+2 \eta \xi}{1+\eta \xi} \frac{u \cdot \tilde{v}}{2 \mu^{2}} \\
& \times\left(\frac{(1+2 \eta \xi)^{2} u^{\mu} u^{v}+2 i \epsilon \xi \tilde{v}^{\mu} u^{v}}{(1+2 \eta \xi)^{2} u^{2}-\xi^{2} \epsilon^{2} v^{2}+i \epsilon \xi(1+2 \eta \xi)(u \cdot \tilde{v})}\right. \\
& +\frac{\xi \mu^{2}}{(1+2 \eta \xi)^{2} u^{2}-\xi^{2} \epsilon^{2} v^{2}+i \epsilon \xi(1+2 \eta \xi)(u \cdot \tilde{v})} \times \\
& \times\left(\delta^{\mu v}+\frac{2(1+2 \eta \xi)^{2} u^{\mu} u^{v}-2 \epsilon^{2} \xi^{2} \tilde{v}^{\mu} \tilde{v}^{v}+i \epsilon \xi(1+2 \eta \xi)\left(u^{\mu} \tilde{v}^{v}+u^{v} \tilde{v}^{\mu}\right)}{(1+2 \eta \xi)^{2} u^{2}-\xi^{2} \epsilon^{2} v^{2}+i \epsilon \xi(1+2 \eta \xi)(u \cdot \tilde{v})}\right. \\
& \left.\left.+\frac{2(1+2 \eta \xi)^{2} u^{\mu} u^{v}-2 \epsilon^{2} \xi^{2} \tilde{v}^{\mu} \tilde{v}^{v}+i \epsilon \xi(1+2 \eta \xi)\left(u^{\mu} \tilde{v}^{v}+u^{v} \tilde{v}^{\mu}\right)}{2 \xi(1+\eta \xi) \mu^{2}}\right)\right) .
\end{aligned}
$$

The singular part of this long expression is in fact quite simple,

$$
\begin{aligned}
\tilde{\Gamma}_{A A}^{(1, d i v)}= & \frac{a g^{2}}{8 \epsilon^{2}} \int \mathrm{d} u \mathrm{~d} v \frac{A_{\mu}(-v) A_{v}(v)}{v^{2}} \int_{1}^{\infty} \frac{\mathrm{d} \xi}{\xi} \frac{\xi-1}{\xi+1} e^{-\xi \frac{u^{2}}{2 \mu^{2}}} \\
& \times \int_{0}^{\infty} \mathrm{d} \eta\left(\delta_{\mu \nu}+2 \frac{\tilde{v}_{\mu} \tilde{v}_{v}}{v^{2}}-\frac{\epsilon^{2} \xi}{1+\eta \xi} \frac{\tilde{v}_{\mu} \tilde{v}_{v}}{\mu^{2}}\right)
\end{aligned}
$$

so in the $\Lambda$-leading order we obtain

$$
\begin{aligned}
\Gamma_{A A}^{(1, d i v)} & =\frac{a g^{2}}{8 \epsilon^{2}} \int \mathrm{d} u \mathrm{~d} v \frac{A^{\mu}(-v) A_{\mu}(v)}{v^{2}} \int_{1}^{\infty} \frac{\mathrm{d} \xi}{\xi} e^{-\xi \frac{u^{2}}{2 \mu^{2}}}, \\
\int_{0}^{\infty} \mathrm{d} \eta & =\frac{a \pi^{3} \mu^{2} g^{2}}{\epsilon^{2}} \beta \Lambda \log \Lambda \int A^{\mu} \square^{-1} A_{\mu} .
\end{aligned}
$$


Adding $\Gamma_{A A}^{(2, d i v)}$ to (4.11) we find

$\Gamma_{A A}^{(d i v)}=-\frac{a \pi^{3} \mu^{2} g^{2}}{\epsilon^{2}} \beta \Lambda \log \Lambda \int \mathrm{d} x A^{\mu}(x) \square^{-1} A_{\mu}(x)$,

which after setting $\beta=1$ becomes

$\Gamma_{A A}^{(d i v)}=-\frac{a \pi^{3} \mu^{2} g^{2}}{\epsilon^{2}} \Lambda \log \Lambda \int A^{\mu} \square^{-1} A_{\mu}$.

\section{Conclusion and outlook}

We calculated in this paper the one-loop corrections to the propagators in a dimensionally reduced Yang-Mills gauge theory defined on the truncated Heisenberg space. The classical action is given by (2.19), (2.20) and the theory is perturbatively quantized around its vacuum solution $\phi=0$, $A_{\mu}=0$. In the previous paper [23] we found the one-loop divergences of the effective action of the first order. They comprise tadpoles

$\int \phi, \int \epsilon^{\mu v} x_{\mu} A_{v}$

and mass terms

$\int \phi \phi, \int A_{\mu} A^{\mu}, \int \epsilon^{\mu v} x_{\mu} A_{\nu} \phi$

Here we calculated the one-loop divergent corrections of the second order to the $\phi \phi$ and $A A$ propagators and found the following additional terms:

$\int \phi \square^{-2} \phi, \quad \int \phi \square^{-1} \phi, \quad \int A_{\mu} \square^{-1} A^{\mu}$.

We have not calculated the $\phi A$ one-loop divergences, but from symmetry we expect that there are nonvanishing nonlocal corrections in this sector too.

The result is not what we expected or hoped for. Namely, in related models with scalar and spinor matter it was possible to attribute renormalizability to the background geometry, that is, to an adequate inclusion of geometric quantities in the Lagrangian [21]. It is well known on the other hand that on commutative curved spaces scalar and spinor theories are renormalizable only if matter is nonminimally coupled to the background curvature and torsion [35], and this pattern is exactly followed in the Grosse-Wulkenhaar and VignesTourneret models. We expected a similar behavior of our noncommutative $U(1)$ model; however, the outcome of our calculation proves differently.
Gauge theories on noncommutative spaces have an additional freedom which comes with the existence of covariant coordinates. This means that one can include the gauge potentials via $\mathbf{X}$ in the action directly, for example as $\left(\mathbf{X}_{\mu} \mathbf{X}^{\mu}\right)^{n}$ or $\exp \left(\alpha_{\mu} \mathbf{X}^{\mu}\right)$, to obtain new classes of theories. Even if one restricts oneself to theories written geometrically, that is, by considering only terms which are proportional to the volume form, there are new gauge-invariant quantities. In our three-dimensional case they are,

$\operatorname{Tr} \mathbf{X}(* \mathbf{X}), \quad \operatorname{Tr} \mathbf{X}^{3}, \quad \operatorname{Tr} \mathbf{X} F, \quad \operatorname{Tr} \mathbf{X}^{2}(* F)$.

However, not all of these expressions are independent because of Eq. (2.8), which on the truncated Heisenberg space reads

$\mathrm{X}^{2}=\mathrm{F}+\mu(* \mathbf{X})-\frac{i \mu^{2}}{4 \epsilon}\left[\theta^{1}, \theta^{2}\right]$

Calculating the first two terms of (5.4) we obtain

$$
\begin{aligned}
\operatorname{Tr} \mathbf{X}(* \mathbf{X})= & \operatorname{Tr}\left(\mathbf{X}_{1}^{2}+\mathbf{X}_{2}^{2}+\left(1-\epsilon^{2}\right) \mathbf{X}_{3}^{2}\right) \\
= & \operatorname{Tr}\left(\frac{\left(1-\epsilon^{2}\right) \mu g}{\epsilon} \phi+\frac{2 \mu^{2} g}{\epsilon} \epsilon^{\mu \nu} x_{\mu} A_{\nu}\right. \\
& \left.-\left(1-\epsilon^{2}\right) g^{2} \phi^{2}-g^{2} A_{\mu} A^{\mu}\right) \\
\operatorname{Tr} \mathbf{X}^{3}= & \operatorname{Tr}\left(\left(3-\epsilon^{2}\right)\left[\mathbf{X}_{1}, \mathbf{X}_{2}\right] \mathbf{X}_{3}+2 i \epsilon \mathbf{X}_{3}\left(\mathbf{X}_{1}^{2}+\mathbf{X}_{2}^{2}\right)\right) \\
= & \operatorname{Tr}\left(\frac{\left(3-\epsilon^{2}\right) \mu^{2} g}{\epsilon} \phi+\frac{2 \mu^{4} g}{\epsilon} x_{\mu} x^{\mu} \phi+\frac{2 \mu^{3} g}{\epsilon} \epsilon^{\mu v} x_{\mu} A_{\nu}\right. \\
- & \left(3-\epsilon^{2}\right) g \phi F_{12}-2 \mu^{2} g^{2} \epsilon^{\mu \nu}\left(x_{\mu} A_{v}+A_{v} x_{\mu}\right) \phi \\
- & \left.\mu g^{2} A_{\mu} A^{\mu}+2 \epsilon g^{3} A_{\mu} A^{\mu} \phi\right)
\end{aligned}
$$

where we neglected the boundary and cosmological terms. The second pair gives

$\operatorname{Tr} \mathrm{XF}=\operatorname{Tr} \mathrm{X}^{3}-\mu \operatorname{Tr} \mathrm{X}(* \mathrm{X})-\operatorname{Tr} \frac{\left(1-\epsilon^{2}\right) \mu^{2} g}{2 \epsilon} \phi$,
$\operatorname{Tr} \mathrm{X}^{2}(* \mathrm{~F})=\operatorname{Tr} \mathrm{F}(* \mathrm{~F})+\mu \operatorname{Tr} \mathrm{XF}-\operatorname{Tr} \frac{\left(1-\epsilon^{2}\right) \mu^{3} g}{2 \epsilon} \phi$.

We see therefore that, were only divergences (5.1), (5.2) present, the theory would have been renormalizable as we could expand the initial action by adding purely geometric terms. It is also interesting to note that addition of the new terms can translate the classical vacuum $\phi=0, A_{\mu}=0$ arbitrarily, which is a point that needs further understanding. But obviously, it is not possible to cancel nonlocal divergences (5.3) in this way, using only polynomial expressions of covariant coordinates. We come again, in this model, 
across an occurrence of the UV/IR mixing. We therefore conclude that geometric gauge theories cannot render a renormalizable theory.

Perhaps a correct way to find a renormalizable gauge model is to consider nonpolynomial interactions, or to add nonlocal terms imposing, as a version of LS duality, symmetry under exchange $\square \leftrightarrow \square^{-1}$. The latter was implemented for the scalar field theory in [13]. Several generalizations were studied for the gauge fields, for example models defined in $[33,34]$ and $[36,37]$; however, the complexity of the actions prevented the complete analysis so it remains unclear which nonlocal operators could render the gauge theory renormalizable. Our present result shows that $\square^{-1}$ terms appear in quantization even in a local version of gauge theory. The other direction of research would be to analyze a matrix model which corresponds to our gauge model. This numerical study could give important information about properties of the gauge fields, and it would then enlarge our understanding of the matrix regularizations.

Finally, a possible explanation of nonrenormalizability of gauge theories is that on noncommutative spaces they are intimately related to gravity. Not only is this seen in the fact that we can combine momenta $p_{\alpha} \in \mathcal{A}$ with the gauge potentials into a unique covariant object. The gauge and coordinate transformations in noncommutative case cannot be clearly separated: indeed, infinitesimal local translations

$\delta \phi=a^{\alpha}\left[p_{\alpha}, \phi\right]$

have the same form as infinitesimal $U(1)$ transformations

$\delta \phi=\epsilon^{\alpha}\left[A_{\alpha}, \phi\right]$,

that is, (5.10) is a special case of (5.11). If gauge theories are a part of gravity or vice versa, then the correct way to understand their renormalizability would be to understand the noncommutative gravity first.

Acknowledgements This work was supported by the Serbian Ministry of Education, Science and Technological Development Grant ON171031.

Open Access This article is distributed under the terms of the Creative Commons Attribution 4.0 International License (http://creativecomm ons.org/licenses/by/4.0/), which permits unrestricted use, distribution, and reproduction in any medium, provided you give appropriate credit to the original author(s) and the source, provide a link to the Creative Commons license, and indicate if changes were made.

Funded by SCOAP ${ }^{3}$.

\section{Appendix 1}

The rest of the contributions - and the respective diagrams - needed for finding the second-order propagator correction $P_{\phi \phi}$ via (3.17) are given now:

$$
\begin{aligned}
& P_{\phi \phi, 12}=-\frac{8 \mu^{4} g^{2}}{(2 \pi)^{8}} \int \mathrm{d} p \mathrm{~d} q \mathrm{~d} k \mathrm{~d} p^{\prime} \mathrm{d} q^{\prime} \mathrm{d} k^{\prime} \delta(p+q+k) \\
& \times \delta\left(p^{\prime}+q^{\prime}+k^{\prime}\right) \frac{p \wedge q}{2} \sin \frac{p \wedge q}{2} \\
& \times \cos \frac{p^{\prime} \wedge q^{\prime}}{2} \frac{1}{(p+q)^{2}} \\
& \times K_{4}\left(r, s \mid p, q, k, p^{\prime}, q^{\prime}, k^{\prime}\right) \\
& +\frac{8 \mu^{2} g^{2}}{(2 \pi)^{6} a} \int \mathrm{d} p \mathrm{~d} q \mathrm{~d} p^{\prime} \mathrm{d} q^{\prime} \delta\left(p+q+p^{\prime}+q^{\prime}\right) \\
& \times \frac{p \wedge q}{2} \sin \frac{p \wedge q}{2} \cos \frac{p^{\prime} \wedge q^{\prime}}{2} \frac{1}{(p+q)^{2}} \\
& \times K_{3}\left(r, s \mid p, q, p^{\prime}, q^{\prime}\right), \\
& P_{\phi \phi, 13}=\frac{8 \epsilon^{2} \mu^{4} g^{2}}{(2 \pi)^{8}} \int \mathrm{d} p \mathrm{~d} q \mathrm{~d} k \mathrm{~d} p^{\prime} \mathrm{d} q^{\prime} \mathrm{d} k^{\prime} \delta(p+q+k) \\
& \times \delta\left(p^{\prime}+q^{\prime}+k^{\prime}\right) \\
& \times \cos \frac{p \wedge q}{2} \cos \frac{p^{\prime} \wedge q^{\prime}}{2} \frac{(p+q)^{\mu}}{(p+q)^{2}} \frac{\partial}{\partial p^{\mu}} \\
& \times K_{4}\left(r, s \mid p, q, k, p^{\prime}, q^{\prime}, k^{\prime}\right) \\
& -\frac{8 \epsilon^{2} \mu^{2} g^{2}}{(2 \pi)^{6} a} \int \mathrm{d} p \mathrm{~d} q \mathrm{~d} p^{\prime} \mathrm{d} q^{\prime} \delta\left(p+q+p^{\prime}+q^{\prime}\right) \\
& \times \cos \frac{p \wedge q}{2} \\
& \times \cos \frac{p^{\prime} \wedge q^{\prime}}{2} \frac{(p+q)^{\mu}}{(p+q)^{2}} \frac{\partial}{\partial p^{\mu}} \\
& \times K_{3}\left(r, s ; p, q, p^{\prime}, q^{\prime}\right),
\end{aligned}
$$

$$
\begin{aligned}
P_{\phi \phi, 15}^{\prime}= & \frac{4 \mu^{4} g^{2}}{(2 \pi)^{6}} \int \mathrm{d} p \mathrm{~d} q \mathrm{~d} p^{\prime} \mathrm{d} q^{\prime} \\
& \times \delta\left(p+q+p^{\prime}+q^{\prime}\right) \\
& \times \frac{p \wedge q}{2} \sin \frac{p \wedge q}{2} \\
& \times \cos \frac{p^{\prime} \wedge q^{\prime}}{2} \frac{1}{p^{2} q^{2}} K_{3}\left(r, s \mid p, q, p^{\prime}, q^{\prime}\right),
\end{aligned}
$$

$$
\begin{aligned}
P_{\phi \phi, 22}= & -\frac{16 \mu^{6} g^{2}}{(2 \pi)^{8} \epsilon^{2}} \int \mathrm{d} p \mathrm{~d} q \mathrm{~d} k \mathrm{~d} p^{\prime} \mathrm{d} q^{\prime} \mathrm{d} k^{\prime} \delta(p+q+k) \\
& \times \delta\left(p^{\prime}+q^{\prime}+k^{\prime}\right) \\
& \times \sin \frac{p \wedge q}{2} \sin \frac{p^{\prime} \wedge q^{\prime}}{2} \frac{(p \wedge q)\left(p^{\prime} \wedge q^{\prime}\right)}{4(p+q)^{2}\left(p^{\prime}+q^{\prime}\right)^{2}} \\
& \times K_{4}\left(r, s \mid p, q, k, p^{\prime}, q^{\prime}, k^{\prime}\right) \\
& -\frac{4 g^{2}}{(2 \pi)^{6} a} \int \mathrm{d} p \mathrm{~d} q \mathrm{~d} p^{\prime} \mathrm{d} q^{\prime} \delta\left(p+q+p^{\prime}+q^{\prime}\right) \\
& \times \sin \frac{p \wedge q}{2} \sin \frac{p^{\prime} \wedge q^{\prime}}{2} \frac{p \cdot p^{\prime}}{(p+q)^{2}} \\
& \times K_{3}\left(r, s \mid p, q, p^{\prime}, q^{\prime}\right)
\end{aligned}
$$




$$
\begin{aligned}
& P_{\phi \phi, 23}=\frac{16 \mu^{6} g^{2}}{(2 \pi)^{8}} \int \mathrm{d} p \mathrm{~d} q \mathrm{~d} k \mathrm{~d} p^{\prime} \mathrm{d} q^{\prime} \mathrm{d} k^{\prime} \delta(p+q+k) \\
& \times \delta\left(p^{\prime}+q^{\prime}+k^{\prime}\right) \\
& \times \cos \frac{p \wedge q}{2} \sin \frac{p^{\prime} \wedge q^{\prime}}{2} \frac{\left(p^{\prime} \wedge q^{\prime}\right)(p+q)^{\mu}}{2(p+q)^{2}\left(p^{\prime}+q^{\prime}\right)^{2}} \frac{\partial}{\partial p^{\mu}} \\
& \times K_{4}\left(r, s \mid p, q, k, p^{\prime}, q^{\prime}, k^{\prime}\right) \\
& \times-\frac{8 \epsilon \mu^{2} g^{2}}{(2 \pi)^{6} a} \int \mathrm{d} p \mathrm{~d} q \mathrm{~d} p^{\prime} \mathrm{d} q^{\prime} \delta\left(p+q+p^{\prime}+q^{\prime}\right) \\
& \times \cos \frac{p \wedge q}{2} \sin \frac{p^{\prime} \wedge q^{\prime}}{2} \frac{\tilde{p}^{\prime \mu}}{(p+q)^{2}} \frac{\partial}{\partial p^{\mu}} \\
& \times K_{3}\left(r, s \mid p, q, p^{\prime}, q^{\prime}\right) \text {, } \\
& P_{\phi \phi, 25}^{\prime}=\frac{8 \mu^{6} g^{2}}{(2 \pi)^{6} \epsilon^{2}} \int \mathrm{d} p \mathrm{~d} q \mathrm{~d} p^{\prime} \mathrm{d} q^{\prime} \\
& \times \delta\left(p+q+p^{\prime}+q^{\prime}\right) \\
& \times \sin \frac{p \wedge q}{2} \sin \frac{p^{\prime} \wedge q^{\prime}}{2} \frac{(p \wedge q)\left(p^{\prime} \wedge q^{\prime}\right)}{4(p+q)^{2} p^{\prime 2} q^{\prime 2}} \\
& \times K_{3}\left(r, s \mid p, q, p^{\prime}, q^{\prime}\right) \text {, } \\
& P_{\phi \phi, 33}=-\frac{16 \epsilon^{2} \mu^{6} g^{2}}{(2 \pi)^{8}} \int \mathrm{d} p \mathrm{~d} q \mathrm{~d} k \mathrm{~d} p^{\prime} \mathrm{d} q^{\prime} \mathrm{d} k^{\prime} \delta(p+q+k) \\
& \times \delta\left(p^{\prime}+q^{\prime}+k^{\prime}\right) \\
& \times \cos \frac{p \wedge q}{2} \cos \frac{p^{\prime} \wedge q^{\prime}}{2} \frac{(p+q)_{\mu}\left(p^{\prime}+q^{\prime}\right)_{\nu}}{(p+q)^{2}\left(p^{\prime}+q^{\prime}\right)^{2}} \\
& \times \frac{\partial^{2}}{\partial p_{\mu} \partial p_{v}^{\prime}} K_{4}\left(r, s \mid p, q, k, p^{\prime}, q^{\prime}, k^{\prime}\right) \\
& -\frac{16 \epsilon^{2} \mu^{4} g^{2}}{(2 \pi)^{6} a} \int \mathrm{d} p \mathrm{~d} q \mathrm{~d} p^{\prime} \mathrm{d} q^{\prime} \\
& \times \delta\left(p+q+p^{\prime}+q^{\prime}\right) \\
& \times \cos \frac{p \wedge q}{2} \cos \frac{p^{\prime} \wedge q^{\prime}}{2} \frac{1}{(p+q)^{2}} \frac{\partial^{2}}{\partial p^{\mu} \partial p_{\mu}^{\prime}} \\
& \times K_{3}\left(r, s \mid p, q, p^{\prime}, q^{\prime}\right) \text {, } \\
& P_{\phi \phi, 35}^{\prime}=\frac{8 \mu^{6} g^{2}}{(2 \pi)^{6}} \int \mathrm{d} p \mathrm{~d} q \mathrm{~d} p^{\prime} \mathrm{d} q^{\prime} \delta\left(p+q+p^{\prime}+q^{\prime}\right) \\
& \times \sin \frac{p \wedge q}{2} \cos \frac{p^{\prime} \wedge q^{\prime}}{2} \frac{(p \wedge q)(p+q)_{\mu}}{2 p^{2} q^{2}(p+q)^{2}} \\
& \times \frac{\partial}{\partial p_{\mu}^{\prime}} K_{3}\left(r, s \mid p, q, p^{\prime}, q^{\prime}\right) \\
& \begin{aligned}
P_{\phi \phi, 45}^{\prime}= & -\frac{8 a \mu^{8} g^{2}}{(2 \pi)^{6} \epsilon^{2}} \int \mathrm{d} p \mathrm{~d} q \mathrm{~d} p^{\prime} \mathrm{d} q^{\prime} \\
& \times \delta\left(p+q+p^{\prime}+q^{\prime}\right) \\
& \times \sin \frac{p \wedge q}{2} \sin \frac{p^{\prime} \wedge q^{\prime}}{2} \frac{(p \wedge q)\left(p^{\prime} \wedge q^{\prime}\right)}{4 p^{2} q^{2} p^{\prime 2}\left(p^{\prime}+q^{\prime}\right)^{2}} \\
& \times K_{3}\left(r, s \mid p, q, p^{\prime}, q^{\prime}\right) \\
& +\frac{2 \mu^{2} g^{2}}{(2 \pi)^{4}} \int \mathrm{d} p \mathrm{~d} q \sin ^{2} \frac{p \wedge q}{2} \frac{1}{p^{2} q^{2}} \\
& \times K_{2}(r, s \mid p+q,-p-q),
\end{aligned} \\
& P_{\phi \phi, 55}^{\prime}=\frac{8 a \mu^{8} g^{2}}{(2 \pi)^{6} \epsilon^{2}} \int \mathrm{d} p \mathrm{~d} q \mathrm{~d} p^{\prime} \mathrm{d} q^{\prime} \delta\left(p+q+p^{\prime}+q^{\prime}\right) \\
& \times \sin \frac{p \wedge q}{2} \\
& \times \sin \frac{p^{\prime} \wedge q^{\prime}}{2} \frac{(p \wedge q)\left(p^{\prime} \wedge q^{\prime}\right)}{4 p^{2} q^{2} p^{\prime 2}\left(p^{\prime}+q^{\prime}\right)^{2}} \\
& \times K_{3}\left(r, s \mid p, q, p^{\prime}, q^{\prime}\right) \\
& +\frac{4 a \mu^{8} g^{2}}{(2 \pi)^{6} \epsilon^{2}} \int \mathrm{d} p \mathrm{~d} q \mathrm{~d} p^{\prime} \mathrm{d} q^{\prime} \\
& \times \delta\left(p+q+p^{\prime}+q^{\prime}\right) \\
& \times \sin \frac{p \wedge q}{2} \sin \frac{p^{\prime} \wedge q^{\prime}}{2} \frac{(p \wedge q)\left(p^{\prime} \wedge q^{\prime}\right)}{4 p^{2} q^{2} p^{\prime 2} q^{\prime 2}} \\
& \times K_{3}\left(r, s \mid p, q, p^{\prime}, q^{\prime}\right) \\
& +\frac{4 \mu^{2} g^{2}}{(2 \pi)^{4}} \int \mathrm{d} p \mathrm{~d} q \sin ^{2} \frac{p \wedge q}{2} \\
& \times\left(-\frac{1}{p^{2} q^{2}}+\frac{p \cdot q}{p^{2} q^{2}(p+q)^{2}}\right. \\
& \left.-\frac{1}{(p+q)^{4}}+\frac{(p \cdot q)^{2}}{p^{2} q^{2}(p+q)^{4}}\right) \\
& \times K_{2}(r, s \mid p+q,-p-q) \text {, } \\
& P_{\phi \phi, 66}=-\frac{4 \mu^{2} g^{2}}{(2 \pi)^{4}} \int \mathrm{d} p \mathrm{~d} q \\
& \times \sin ^{2} \frac{p \wedge q}{2}\left(\frac{1}{(p+q)^{4}}-\frac{(p \cdot q)^{2}}{p^{2} q^{2}(p+q)^{4}}\right) \\
& \times K_{2}(r, s \mid p+q,-p-q) \text {. }
\end{aligned}
$$


They correspond to the additional diagrams:

$+\frac{2 \mu^{2} g^{2}}{(2 \pi)^{4}} \int \mathrm{d} p \mathrm{~d} q \sin ^{2} \frac{p \wedge q}{2}$
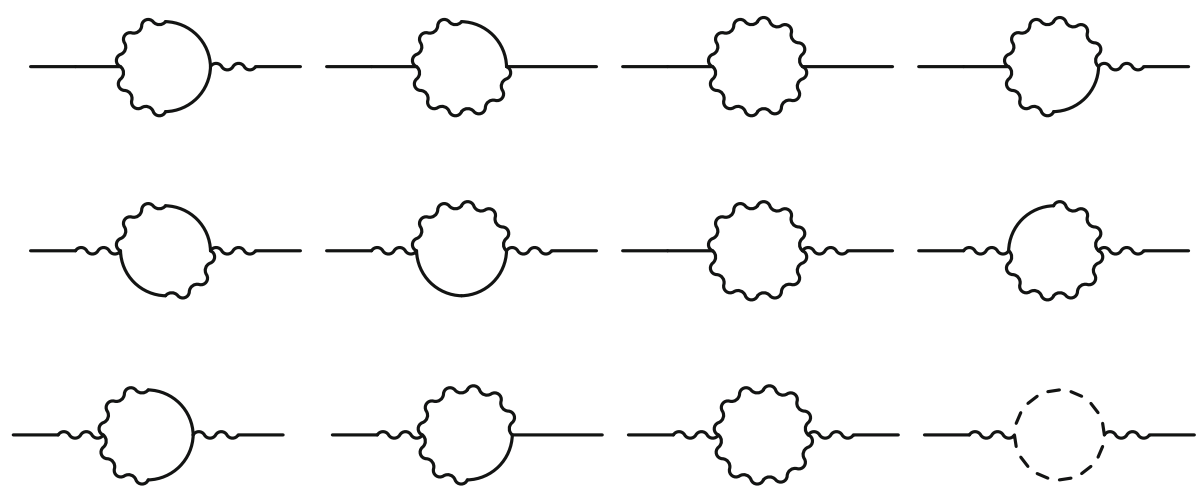

The dashed line is the ghost propagator $\bar{c} c$. The oneloop propagator corrections $P_{\phi \phi, 7}, P_{\phi \phi, 8}, P_{\phi \phi, 9}$, and $P_{\phi \phi, 10}$, which were calculated in [23], are

$$
\begin{aligned}
& P_{\phi \phi, 7}=\frac{2 \mu^{2} g^{2}}{(2 \pi)^{6}} \int \mathrm{d} p \mathrm{~d} q \mathrm{~d} p^{\prime} \mathrm{d} q^{\prime} \delta\left(p+q+p^{\prime}+q^{\prime}\right) \\
& \times \sin \frac{p \wedge q}{2} \sin \frac{p^{\prime} \wedge q^{\prime}}{2} \frac{p \cdot p^{\prime}}{p^{2} p^{\prime 2}} K_{3}\left(r, s \mid p, q, p^{\prime}, q^{\prime}\right) \\
& +\frac{4 g^{2}}{(2 \pi)^{4} a} \int \mathrm{d} p \mathrm{~d} q \\
& \times \sin ^{2} \frac{p \wedge q}{2} \frac{1}{p^{2}} K_{2}(r, s \mid q,-q), \\
& P_{\phi \phi, 8}=\frac{2 \epsilon^{2} \mu^{2} g^{2}}{(2 \pi)^{6}} \int \mathrm{d} p \mathrm{~d} q \mathrm{~d} p^{\prime} \mathrm{d} q^{\prime} \delta\left(p+q+p^{\prime}+q^{\prime}\right) \\
& \times \cos \frac{p \wedge q}{2} \cos \frac{p^{\prime} \wedge q^{\prime}}{2} \frac{p \cdot p^{\prime}}{p^{2} p^{\prime 2}} K_{3}\left(r, s \mid p, q, p^{\prime}, q^{\prime}\right) \\
& +\frac{4 \epsilon^{2} g^{2}}{(2 \pi)^{4} a} \int \mathrm{d} p \mathrm{~d} q \\
& \times \cos ^{2} \frac{p \wedge q}{2} \frac{1}{p^{2}} K_{2}(r, s \mid q,-q), \\
& P_{\phi \phi, 9}=-\frac{4 \mu^{4} g^{2}}{(2 \pi)^{6}} \int \mathrm{d} p \mathrm{~d} q \mathrm{~d} p^{\prime} \mathrm{d} q^{\prime} \delta\left(p+q+p^{\prime}+q^{\prime}\right) \\
& \times \sin \frac{p \wedge q}{2} \cos \frac{p^{\prime} \wedge q^{\prime}}{2} \frac{p \wedge q}{2 p^{2} q^{2}} \\
& \times K_{3}\left(r, s \mid p, q, p^{\prime}, q^{\prime}\right) \text {, } \\
& P_{\phi \phi, 10}=-\frac{2 a \mu^{8} g^{2}}{(2 \pi)^{6} \epsilon^{2}} \int \mathrm{d} p \mathrm{~d} q \mathrm{~d} p^{\prime} \mathrm{d} q^{\prime} \\
& \times \delta\left(p+q+p^{\prime}+q^{\prime}\right) \\
& \times \sin \frac{p \wedge q}{2} \sin \frac{p^{\prime} \wedge q^{\prime}}{2} \\
& \times \frac{(p \wedge q)\left(p^{\prime} \wedge q^{\prime}\right)}{4 p^{2} q^{2} p^{\prime 2} q^{\prime 2}} K_{3}\left(r, s \mid p, q, p^{\prime}, q^{\prime}\right)
\end{aligned}
$$

The ghost contributions are zero.

\section{Appendix 2}

Some of the Gaussian integrals in two dimensions are

$$
\begin{aligned}
\int \mathrm{d} p e^{-a p^{2}+b \cdot p} & =\frac{\pi}{a} e^{b^{2} / 4 a} \\
\int \mathrm{d} p p_{\alpha} p_{\beta} e^{-a p^{2}+b \cdot p} & =\frac{\pi}{2 a^{2}}\left(\delta_{\alpha \beta}+\frac{b_{\alpha} b_{\beta}}{2 a}\right) e^{b^{2} / 4 a}, \\
\int \mathrm{d} p\left(p^{2}\right)^{2} e^{-a p^{2}+b \cdot p} & =\frac{\pi}{a^{3}}\left(2+\frac{b^{2}}{a}+\frac{\left(b^{2}\right)^{2}}{16 a^{2}}\right) e^{b^{2} / 4 a} \\
\int \mathrm{d} p \frac{p_{\alpha} p_{\beta}}{p^{2}} e^{-a p^{2}+b \cdot p} & =\frac{2 \pi}{b^{2}}\left(\frac{b^{2} \delta_{\alpha \beta}-2 b_{\alpha} b_{\beta}}{b^{2}}\right. \\
& \left.+\frac{b_{\alpha} b_{\beta}}{2 a}\right) e^{b^{2} / 4 a} .
\end{aligned}
$$

Switching from $u$-integration to $u^{2}$-integration:

$$
\begin{aligned}
& \int \mathrm{d} u f\left(u^{2}\right) \frac{u_{\alpha} u_{\beta}}{u^{2}}=\frac{\pi}{2} \delta_{\alpha \beta} \int \mathrm{d}\left(u^{2}\right) f\left(u^{2}\right) \\
& \quad \times \int \mathrm{d} u f\left(u^{2}\right) \frac{u_{\alpha} u_{\beta} u_{\gamma} u_{\delta}}{\left(u^{2}\right)^{2}} \\
& =\frac{\pi}{8}\left(\delta_{\alpha \beta} \delta_{\gamma \delta}+\delta_{\alpha \gamma} \delta_{\beta \delta}+\delta_{\alpha \delta} \delta_{\beta \gamma}\right) \int \mathrm{d}\left(u^{2}\right) f\left(u^{2}\right)
\end{aligned}
$$

Some formulas used to evaluate the amputated propagators are

$\frac{1}{(2 \pi)^{4}} \int \mathrm{d} u K_{2}(p, q, k, u) K^{-1}(-u, r)$ 
672 Page 14 of 17

Eur. Phys. J. C (2016) 76:672

$$
\begin{aligned}
= & \delta(p+r) K(q, k)+\delta(q+r) \\
& \times K(p, q)+\delta(k+r) K(p, q) \\
& \times \frac{1}{(2 \pi)^{8}} \int \mathrm{d} u \mathrm{~d} v K^{-1}(r,-u) K_{3}\left(u, v \mid p, q, p^{\prime}, q^{\prime}\right) \\
& \times K^{-1}(-u, r) \\
= & \delta(p+r) \delta(q+s) K\left(p^{\prime}, q^{\prime}\right)+\delta(p+r) \\
& \times \delta\left(p^{\prime}+s\right) K\left(q, q^{\prime}\right)+\delta(p+r) \delta\left(q^{\prime}+s\right) K\left(q, p^{\prime}\right) \\
& +\delta(q+r) \delta(p+s) K\left(p^{\prime}, q^{\prime}\right)+\delta(q+r) \\
& \times \delta\left(q^{\prime}+s\right) K\left(p, p^{\prime}\right)+\delta(q+r) \delta\left(p^{\prime}+s\right) K(p, q) \\
& +(r \leftrightarrow s) .
\end{aligned}
$$

Appendix 3

Divergent contributions to the $\phi \phi$-part of the effective action after expansion around $u=0$ are

$$
\begin{aligned}
& \Gamma_{\phi \phi}^{(2)}=-\frac{3 \mu^{2} a g^{2}}{\epsilon^{2}} \int \mathrm{d} u \mathrm{~d} v \frac{\phi(-v) \phi(v)}{\left(v^{2}\right)^{2}} \\
& \times \int_{1}^{\infty} \frac{\mathrm{d} \xi}{\xi} \frac{\xi-1}{\xi+1} e^{-\xi \frac{u^{2}}{2 \mu^{2}}} \int_{0}^{\infty} \mathrm{d} \eta e^{-\frac{\xi \epsilon^{2}}{1+\eta \xi} \frac{v^{2}}{2 \mu^{2}}} \\
& -a g^{2} \int \mathrm{d} u \mathrm{~d} v \frac{\phi(-v) \phi(v)}{v^{2}} \\
& \times \int_{1}^{\infty} \mathrm{d} \xi \frac{\xi-1}{\xi+1} e^{-\xi \frac{u^{2}}{2 \mu^{2}}} \int_{0}^{\infty} \frac{\mathrm{d} \eta}{1+\eta \xi} e^{-\frac{\xi \epsilon^{2}}{1+\eta \xi} \frac{v^{2}}{2 \mu^{2}}} \\
& +\frac{a g^{2}}{2} \int \mathrm{d} u \mathrm{~d} v \frac{\phi(-v) \phi(v)}{v^{2}} \\
& \times \int_{1}^{\infty} \mathrm{d} \xi \frac{\xi-1}{\xi+1} e^{-\xi \frac{u^{2}}{2 \mu^{2}}} \int_{0}^{\infty} \frac{\mathrm{d} \eta}{(1+2 \eta \xi)^{2}-\epsilon^{2} \xi^{2}} \\
& \times\left(2-\frac{1+\epsilon^{2} \xi^{2}}{1+\eta \xi}-\frac{2 \epsilon^{2} \xi^{3}}{(1+2 \eta \xi)^{2}-\epsilon^{2} \xi^{2}} \frac{\epsilon^{2} \xi+\eta}{1+\eta \xi}\right), \\
& \Gamma_{\phi \phi}^{(3)}=\frac{\mu^{2} g^{2}}{\epsilon^{2}} \int \mathrm{d} u \mathrm{~d} v \frac{\phi(-v) \phi(v)}{\left(v^{2}\right)^{2}} \\
& \times \int_{1}^{\infty} \frac{\mathrm{d} \xi}{\xi} \frac{\xi-1}{\xi+1} e^{-\xi \frac{u^{2}}{2 \mu^{2}}} \int_{0}^{\infty} \mathrm{d} \eta e^{-\frac{\xi \epsilon^{2}}{1+\eta \xi} \frac{v^{2}}{2 \mu^{2}}} \\
& +\mu^{2} g^{2} \int \mathrm{d} u \mathrm{~d} v \frac{\phi(-v) \phi(v)}{\left(v^{2}\right)^{2}} \\
& \times \int_{1}^{\infty} \mathrm{d} \xi \xi \frac{\xi-1}{\xi+1} e^{-\xi \frac{u^{2}}{2 \mu^{2}}} \int_{0}^{\infty} \mathrm{d} \eta \frac{(1+2 \eta \xi)^{2}+\epsilon^{2} \xi^{2}}{\left((1+2 \eta \xi)^{2}-\epsilon^{2} \xi^{2}\right)^{2}} \\
& -2 \epsilon^{2} \mu^{2} g^{2} \int \frac{\mathrm{d} u}{u^{2}} \mathrm{~d} v \frac{\phi(-v) \phi(v)}{v^{2}} \\
& \times \int_{1}^{\infty} \mathrm{d} \xi \xi^{3} \frac{\xi-1}{\xi+1} e^{-\xi \frac{u^{2}}{2 \mu^{2}}} \int_{0}^{\infty} \frac{\mathrm{d} \eta}{\left((1+2 \eta \xi)^{2}-\epsilon^{2} \xi^{2}\right)^{2}}
\end{aligned}
$$

$$
\begin{aligned}
& -g^{2} \int \mathrm{d} u \mathrm{~d} v \frac{\phi(-v) \phi(v)}{v^{2}} \\
& \times \int_{1}^{\infty} \mathrm{d} \xi \frac{\xi-1}{\xi+1} e^{-\xi \frac{u^{2}}{2 \mu^{2}}} \int_{0}^{\infty} \mathrm{d} \eta \frac{(1+2 \eta \xi)^{2}}{(1+2 \eta \xi)^{2}-\epsilon^{2} \xi^{2}} \\
& +\frac{g^{2}}{2} \int \mathrm{d} u \mathrm{~d} v \frac{\phi(-v) \phi(v)}{v^{2}} \\
& \times \int_{1}^{\infty} \mathrm{d} \xi \frac{\xi-1}{\xi+1} e^{-\xi \frac{u^{2}}{2 \mu^{2}}} \int_{0}^{\infty} \frac{\mathrm{d} \eta}{1+\eta \xi} \frac{(1+2 \eta \xi)^{2}+\epsilon^{2} \xi^{2}}{(1+2 \eta \xi)^{2}-\epsilon^{2} \xi^{2}} \\
& +\epsilon^{2} g^{2} \int \mathrm{d} u \mathrm{~d} v \frac{\phi(-v) \phi(v)}{v^{2}} \\
& \times \int_{1}^{\infty} \mathrm{d} \xi \xi^{3} \frac{\xi-1}{\xi+1} e^{-\xi \xi \frac{u^{2}}{2 \mu^{2}}} \int_{0}^{\infty} \frac{\mathrm{d} \eta}{1+\eta \xi} \frac{\epsilon^{2} \xi+\eta}{\left((1+2 \eta \xi)^{2}-\epsilon^{2} \xi^{2}\right)^{2}},
\end{aligned}
$$

$$
\begin{aligned}
\Gamma_{\phi \phi}^{(4)}= & a \mu^{2} g^{2} \int \mathrm{d} u \mathrm{~d} v \frac{\phi(-v) \phi(v)}{\left(v^{2}\right)^{2}} \\
& \times \int_{1}^{\infty} \frac{\mathrm{d} \xi}{\xi} \frac{\xi-1}{\xi+1} e^{-\xi \frac{u^{2}}{2 \mu^{2}}} \int_{0}^{\infty} \frac{\mathrm{d} \eta}{\eta^{2}} e^{-\frac{\eta}{1+\eta \xi} \frac{v^{2}}{2 \mu^{2}}} \\
& -a \mu^{2} g^{2} \int \mathrm{d} u \mathrm{~d} v \frac{\phi(-v) \phi(v)}{\left(v^{2}\right)^{2}} \\
& \times \int_{1}^{\infty} \frac{\mathrm{d} \xi}{\xi} \frac{\xi-1}{\xi+1} e^{-\xi \frac{u^{2}}{2 \mu^{2}} \int_{0}^{\infty} \mathrm{d} \eta \frac{\eta^{2}+\epsilon^{2}}{\left(\eta^{2}-\epsilon^{2}\right)^{2}} e^{-\frac{\eta+\xi \epsilon^{2}}{1+\eta \xi} \frac{v^{2}}{2 \mu^{2}}}} \\
& +a \epsilon^{2} g^{2} \int \mathrm{d} u \mathrm{~d} v \frac{\phi(-v) \phi(v)}{v^{2}} \\
& \times \int_{1}^{\infty} \mathrm{d} \xi \frac{\xi-1}{\xi+1} e^{-\xi \frac{u^{2}}{2 \mu^{2}}} \int_{0}^{\infty} \frac{\mathrm{d} \eta}{\left(\eta^{2}-\epsilon^{2}\right)(1+\eta \xi)} e^{-\frac{\eta+\xi \epsilon^{2}}{1+\eta \xi} \frac{v^{2}}{2 \mu^{2}}} .
\end{aligned}
$$

Appendix 4

4.1 Relation between the $\xi$-integral and the $u^{2}$-divergence

As seen in the formulas throughout the paper, the integrals over $\xi$ contain an exponent $\lambda=u^{2} / 2 \mu^{2}$, which regularizes them at the upper bound

$\int_{1}^{\infty} \mathrm{d} \xi f(\xi) e^{-\lambda \xi}$

This exponent is lost if we expand in small $u^{2}$ by putting $u^{2}=0$ : in that case the IR divergence in $u$ is transferred to divergence in $\xi$. For better control, we will keep the exponent.

The encountered integrals can be written as

$$
\int_{1}^{\infty} \mathrm{d} \xi f(\xi) e^{-\lambda \xi}=\int_{1}^{\infty} \mathrm{d} \xi g(\xi) h(1 / \xi) e^{-\lambda \xi},
$$

Springer 
where $g(\xi)$ is a simpler function of $\xi$ and $h(\xi)$ has the form

$h(\xi)=H+O(1 / \xi), \quad H=$ const.

Let us analyze two integrals

$$
\begin{aligned}
I_{1}(\lambda) & =\int_{1}^{\infty} \mathrm{d} \xi g(\xi) e^{-\lambda \xi}, \quad I_{2}(\lambda)=\int_{1}^{\infty} \mathrm{d} \xi \frac{g(\xi)}{\xi} e^{-\lambda \xi}, \\
\frac{\mathrm{d} I_{2}}{\mathrm{~d} \lambda} & =-I_{1} .
\end{aligned}
$$

The last equation implies that if $I_{2}=O\left(\lambda^{n}\right)$ when $\lambda \rightarrow 0$, then $I_{1}=O\left(\lambda^{n-1}\right)$ and

$$
\frac{I_{2}}{I_{1}} \rightarrow 0, \quad \lambda \rightarrow 0
$$

In other words if both $I_{1}$ and $I_{2}$ are divergent, then the $I_{1}$ divergence is of the higher order. Since we are interested in the leading divergence in $\lambda$, we can discard the $O(1 / \xi)$ contribution in (9.3) and simplify the initial integral:

$$
\int_{1}^{\infty} \mathrm{d} \xi f(\xi) e^{-\lambda \xi} \longrightarrow H \int_{1}^{\infty} \mathrm{d} \xi g(\xi) e^{-\lambda \xi}
$$

The actual simplified integrals appearing in the effective action are the following $(n>0, \gamma$ is the Euler-Mascheroni constant):

$$
\begin{gathered}
\int_{1}^{\infty} \mathrm{d} \xi \xi^{n} e^{-\lambda \xi}=\frac{n !}{\lambda^{n+1}}-\frac{1}{n+1}+O(\lambda), \\
\int_{1}^{\infty} \frac{\mathrm{d} \xi}{\xi} e^{-\lambda \xi}=-\log \lambda-\gamma+O(\lambda), \\
\int_{1}^{\infty} \frac{\mathrm{d} \xi}{\xi n} e^{-\lambda \xi}=\frac{1}{n}+O(\lambda), \\
\int_{1}^{\infty} \mathrm{d} \xi \frac{\log \xi}{\xi} e^{-\lambda \xi}= \\
=\frac{1}{2} \log ^{2} \lambda+\gamma \log \lambda \\
+\frac{\gamma^{2}}{2}+\frac{\pi^{2}}{12}+O(\lambda) .
\end{gathered}
$$

\subsection{Detailed analysis}

Expansion around $u=0$ gives, besides (4.6), further terms listed in Appendix 3 which are potentially divergent at the lower boundary of integration in $u$. The selected terms contain

$$
\int \mathrm{d} v \frac{\phi(-v) \phi(v)}{\left(v^{2}\right)^{2}}, \quad \int \mathrm{d} v \frac{\phi(-v) \phi(v)}{v^{2}},
$$

which give nonlocal contributions to the one-loop effective action,

$$
\int \phi \square^{-2} \phi, \quad \int \phi \square^{-1} \phi
$$

All expressions contain integrations over two parameters: we wish to sum divergent contributions and see weather the result is zero, finite or divergent. We first observe the exponentials in $v^{2}$ in $\Gamma_{\phi \phi}^{(2)}$ and $\Gamma_{\phi \phi}^{(4)}$. In order to extract the $\square^{-1}$ and $\square^{-2}$-parts of the one-loop effective action, we expand this exponential in power series and consider only the first two terms: the remaining ones give local contributions.

To explain the regularization procedure, we start with the integral

$$
\begin{aligned}
I= & \int_{0} \mathrm{~d} u \mathrm{~d} v \frac{\phi(-v) \phi(v)}{\left(v^{2}\right)^{2}} \int_{1}^{\infty} \frac{\mathrm{d} \xi}{\xi} \frac{\xi-1}{\xi+1} e^{-\frac{\xi u^{2}}{2 \mu^{2}}} \\
& \times \int_{0}^{\infty} \mathrm{d} \eta e^{-\frac{\epsilon^{2} \xi}{1+\eta \frac{\eta^{2}}{2}}} \frac{v^{2}}{2 \mu^{2}}
\end{aligned}
$$

We introduce regularizations in the $u$ - and in the $\eta$-integrals. We choose the regulators to be defined by the same large parameter $\Lambda$ :

$$
\begin{array}{r}
\int \mathrm{d} u f\left(u^{2}\right) \longrightarrow \pi \int_{\mu^{2} / \Lambda} d\left(u^{2}\right) f\left(u^{2}\right), \\
\int \mathrm{d} \eta \longrightarrow \int_{\beta_{2} / \Lambda}^{\beta \Lambda} \mathrm{d} \eta .
\end{array}
$$

We find

$$
\begin{aligned}
I^{(d i v)}= & \pi \int_{\mu^{2} / \Lambda} \mathrm{d} v \frac{\phi(-v) \phi(v)}{\left(v^{2}\right)^{2}} \int_{\mu_{2}} d\left(u^{2}\right) \\
& \times \int_{1}^{\infty} \frac{\mathrm{d} \xi}{\xi} \frac{\xi-1}{\xi+1} e^{-\frac{\xi u^{2}}{2 \mu^{2}}} \\
& \times \int_{\beta \Lambda}^{\beta \Lambda} \mathrm{d} \eta\left(1-\frac{\epsilon^{2} \xi}{1+\eta \xi} \frac{v^{2}}{2 \mu^{2}}\right) .
\end{aligned}
$$

We can set the lower boundary of the integral over $\eta$ to zero since it contains no divergence. Integration over $\eta$ and Fourier transformation give 


$$
\begin{aligned}
I^{(d i v)}= & 4 \pi^{3}\left(\beta \Lambda \int \phi \square^{-2} \phi \int_{\mu^{2} / \Lambda} d\left(u^{2}\right)\right. \\
& \times \int_{1}^{\infty} \frac{\mathrm{d} \xi}{\xi} \frac{\xi-1}{\xi+1} e^{-\frac{\xi u^{2}}{2 \mu^{2}}} \\
& -\frac{\epsilon^{2}}{2 \mu^{2}} \int \phi \square^{-1} \phi \int_{\mu^{2} / \Lambda} d\left(u^{2}\right) \\
& \left.\times \int_{1}^{\infty} \frac{\mathrm{d} \xi}{\xi} \frac{\xi-1}{\xi+1} \log (\beta \Lambda \xi+1) e^{-\frac{\xi u^{2}}{2 \mu^{2}}}\right) .
\end{aligned}
$$

In accordance with the previous discussion leading to (9.6), we keep only the leading contribution at $\xi \rightarrow \infty$ and obtain

$$
\begin{aligned}
I^{(d i v)}= & 4 \pi^{3}\left(\beta \Lambda \int \phi \square^{-2} \phi \int_{\mu^{2} / \Lambda} d\left(u^{2}\right)\right. \\
& \times \int_{1}^{\infty} \frac{\mathrm{d} \xi}{\xi} e^{-\frac{\xi u^{2}}{2 \mu^{2}}} \\
& -\frac{\epsilon^{2}}{2 \mu^{2}} \int \phi \square^{-1} \phi \int_{\mu^{2} / \Lambda} d\left(u^{2}\right) \\
& \left.\times \int_{1}^{\infty} \frac{\mathrm{d} \xi}{\xi}(\log \Lambda+\log \xi) e^{-\frac{\xi u^{2}}{2 \mu^{2}}}\right) .
\end{aligned}
$$

The remaining integration gives the following leading contributions in $\Lambda$ :

$$
\begin{aligned}
I^{(d i v)}= & 4 \pi^{3} \mu^{2}\left(-\beta \log \Lambda \int \phi \square^{-2} \phi\right. \\
& \left.+\frac{3 \epsilon^{2}}{4 \mu^{2}} \frac{\log ^{2} \Lambda}{\Lambda} \int \phi \square^{-1} \phi\right) .
\end{aligned}
$$

The second term vanishes for $\Lambda \rightarrow \infty$.

Inspecting the other terms given in Appendix 3 we see that some of the integrals have singular points which are inside the integration domain. In such cases the regulators are introduced in the following manner. Let $\zeta=\xi, \eta$ denote the integration parameter and $\zeta=\zeta_{0}$ the pole of the integration function. We regularize as before using the same regulator $\Lambda$, replacing

$$
\int \mathrm{d} \zeta \rightarrow \int^{\zeta_{0}-\frac{\alpha}{\Lambda}} \mathrm{d} \zeta+\int_{\zeta_{0}+\frac{\alpha}{\Lambda}} \mathrm{d} \zeta
$$

where $\alpha$ is a positive constant. Concretely, we make the following substitutions:
- for $\zeta=\eta$ and the pole arising from $\eta^{2}-\epsilon^{2}=0$, we denote $\alpha=\gamma$,

- for $\zeta=\eta$ and the pole arising from $(1+2 \eta \xi)^{2}-\epsilon^{2} \xi^{2}=$ $0, \alpha=\gamma_{2}$,

- for $\zeta=\xi$ and the pole arising from $\log \mid(1-\epsilon \xi) /(1+$ $\epsilon \xi) \mid=\infty, \alpha=\delta$.

We regularize all integrals given in Appendix 3. Calculating them and adding different contributions, we find that the leading-order propagator divergences of the one-loop effective action are

$$
\begin{aligned}
& -4 \pi^{3} \mu^{4}\left(\frac{1-3 a}{\epsilon^{2}} \beta+\frac{a}{\beta_{2}}-\frac{a}{\gamma}+\frac{1+a}{4 \gamma_{2}}\right) \\
& \times \log \Lambda \int \phi \square^{-2} \phi
\end{aligned}
$$

and

$$
-\frac{\epsilon^{2} \pi^{3} \mu^{2}}{2 \gamma_{2}} \Lambda \log ^{2} \Lambda \int \phi \square^{-1} \phi .
$$

An additional divergent $\square^{-1}$-term comes from the gauge vertices with no Mehler kernel. It is quadratic in $\Lambda$ and equal to

$\frac{\epsilon^{2} \pi^{3} \mu^{2}}{\beta_{2}^{2}} \Lambda^{2} \int \phi \square^{-1} \phi$.

Our results contain yet undefined parameters $\beta, \beta_{2}, \gamma, \gamma_{2}$. They were introduced to examine the possibility to cancel divergences by an appropriate choice of the regulators. This method of course is a kind of fine tuning, since one really does not wish to introduce a large number of different regulators. We, however, find that in any case it is impossible to remove the $\square^{-1}$ divergence: since $\beta_{2} \neq \infty$, divergence in (9.14) always remains. On the other hand, divergent $\square^{-2}$ term (9.12) can be removed for some values of $a$ by an appropriate choice of $\beta, \beta_{2}, \gamma, \gamma_{2}$; however, in the non-propagating case $a=$ 0 , the term remains. In the light of this, we shall set the parameters to the simplest value

$\beta=\beta_{2}=\gamma=\gamma_{2}=1$,

with which the leading one-loop $\phi \phi$-propagator divergences become

$$
\left(\frac{8}{\epsilon^{2}}-14+\epsilon^{2}\right) \pi^{3} \mu^{4} g^{2} \log \Lambda \int \phi \square^{-2} \phi
$$

and

$$
\epsilon^{2} \pi^{3} \mu^{2} g^{2} \Lambda^{2} \int \phi \square^{-1} \phi
$$

\section{References}

1. M.R. Douglas, N.A. Nekrasov, Rev. Mod. Phys. 73, 977 (2001). doi:10.1103/RevModPhys.73.977. arXiv:hep-th/0106048 
2. C.S. Chu, J. Madore, H. Steinacker, JHEP 0108, 038 (2001). doi:10. 1088/1126-6708/2001/08/038. arXiv:hep-th/0106205

3. H. Grosse, R. Wulkenhaar, JHEP 0312, 019 (2003). arXiv:hep-th/0307017

4. H. Grosse, R. Wulkenhaar, Commun. Math. Phys. 256, 305 (2005). arXiv:hep-th/0401128

5. E. Langmann, R.J. Szabo, Phys. Lett. B 533, 168 (2002). arXiv:hep-th/0202039

6. R. Gurau, J. Magnen, V. Rivasseau, F. Vignes-Tourneret, Commun. Math. Phys. 267, 515 (2006). arXiv:hep-th/0512271

7. V. Rivasseau, F. Vignes-Tourneret, R. Wulkenhaar, Commun. Math. Phys. 262, 565-594 (2006)

8. H. Grosse, R. Wulkenhaar, Eur. Phys. J. C 35, 277 (2004). arXiv:hep-th/0402093

9. M. Disertori, R. Gurau, J. Magnen, V. Rivasseau, Phys. Lett. B 649, 95 (2007). arXiv:hep-th/0612251

10. H. Grosse, R. Wulkenhaar, J. Geom. Phys. 62, 1583 (2012). arXiv:0709.0095 [hep-th]

11. F. Vignes-Tourneret, Annales Henri Poincare 8, 427 (2007). arXiv:math-ph/0606069

12. D.J. Gross, A. Neveu, Phys. Rev. D 10, 3235 (1974)

13. R. Gurau, J. Mangen, V. Rivasseau, A. Tanasa, Commun. Math. Phys. 287, 275-290 (2009). arXiv:0802.0791

14. D.N. Blaschke, E. Kronberger, R.I.P. Sedmik, M. Wohlgenannt, SIGMA 6, 06 (2010). arXiv:1004.2127 [hep-th]

15. D.N. Blaschke, H. Grosse, M. Schweda, Europhys. Lett. 79, 61002 (2007). arXiv:0705.4205 [hep-th]

16. A. de Goursac, J.C. Wallet, R. Wulkenhaar, Eur. Phys. J. C 51, 977 (2007). arXiv:hep-th/0703075 [HEP-TH]

17. H. Grosse, M. Wohlgenannt, Eur. Phys. J. C 52, 435 (2007). arXiv:hep-th/0703169

18. J. Madore, S. Schraml, P. Schupp, J. Wess, Eur. Phys. J. C 16, 161 (2000). arXiv:hep-th/0001203

19. P. Martinetti, P. Vitale, J.C. Wallet, JHEP 1309, 051 (2013). arXiv:1303.7185 [hep-th]
20. M. Buric, M. Wohlgenannt, JHEP 1003, 053 (2010). arXiv:0902.3408 [hep-th]

21. M. Buric, J. Madore, L. Nenadovic. arXiv:1502.00761 [hep-th]

22. M. Buric, H. Grosse, J. Madore, JHEP 1007, 010 (2010). arXiv:1003.2284 [hep-th]

23. M. Buric, M. Dimitrijevic, V. Radovanovic, M. Wohlgenannt, Phys. Rev. D 86, 105024 (2012)

24. M. Buric, J. Madore, PoS QGQGS 2011, 010 (2011)

25. J. Madore, An introduction to noncommutative differential geometry and its physical applications. Lond. Math. Soc. Lect. Note Ser. 257, 1 (2000)

26. E. Kronberger, Models with oscillator terms in noncommutative quantum field theory. Ph.D. Thesis, Technical University of Vienna, 2010

27. L. Nenadovic, PhD Thesis. University of Belgrade, 2016

28. L. Nenadovic, D. Prekrat, (In preparation)

29. M. Hayakawa. arXiv:hep-th/9912167

30. M. Hayakawa, Phys. Lett. B 478, 394 (2000). doi:10.1016/ S0370-2693(00)00242-2. arXiv:hep-th/9912094

31. F.R. Ruiz, Phys. Lett. B 502, 274 (2001). doi:10.1016/ S0370-2693(01)00145-9. arXiv:hep-th/0012171

32. M. Attems, D.N. Blaschke, M. Ortner, M. Schweda, S. Stricker, M. Weiretmayr, JHEP 0507, 071 (2005). arXiv:hep-th/0506117

33. D.N. Blaschke, F. Gieres, E. Kronberger, M. Schweda, M. Wohlgenannt, J. Phys. A 41, 252002 (2008). arXiv:0804.1914 [hep-th]

34. D.N. Blaschke, A. Rofner, M. Schweda, R.I.P. Sedmik, Eur. Phys. J. C 62, 433 (2009). doi:10.1140/epjc/s10052-009-1031-1. arXiv:0901.1681 [hep-th]

35. I.L. Shapiro, Phys. Rep. 357, 113 (2002). doi:10.1016/ S0370-1573(01)00030-8. arXiv:hep-th/0103093

36. D.N. Blaschke, A. Rofner, R.I.P. Sedmik, M. Wohlgenannt, J. Phys. A 43, 425401 (2010). doi:10.1088/1751-8113/43/42/ 425401. arXiv:0912.2634 [hep-th]

37. D.N. Blaschke, Europhys. Lett. 91, 11001 (2010). doi:10.1209/ 0295-5075/91/11001. arXiv:1005.1578 [hep-th] 